Review Article

\title{
Aspirin use for cancer prevention: A systematic review of public, patient and healthcare provider attitudes and adherence behaviours
}

\author{
Kelly E. Lloyd ${ }^{\text {a, }}$, Louise H. Hall ${ }^{\mathrm{a}}$, Natalie King ${ }^{\mathrm{a}}$, Rachael J. Thorneloe ${ }^{\mathrm{b}}$, \\ Rocio Rodriguez-Lopez ${ }^{\mathrm{a}}$, Lucy Ziegler ${ }^{\mathrm{a}}$, David G. Taylor ${ }^{\mathrm{c}}$, Mairead MacKenzie ${ }^{\mathrm{d}}$, \\ Samuel G. Smith ${ }^{\text {a }}$, on behalf of the AsCaP Group

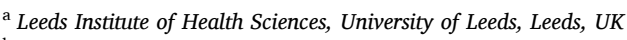 \\ ${ }^{\mathrm{b}}$ Centre for Behavioural Science \& Applied Psychology, Sheffield Hallam University, Sheffield, UK \\ ${ }^{c}$ School of Pharmacy, University College London, London, UK \\ 'Independent Cancer Patients' Voice, UK
}

\section{A R T I C L E I N F O}

\section{Keywords:}

Preventive therapy

Chemoprevention

Decision-making

Aspirin

NSAID

\begin{abstract}
A B S T R A C T
We undertook a systematic review to synthesise the data on attitudes and behaviour towards the use of aspirin for cancer prevention, and healthcare providers' attitudes towards implementing aspirin in practice. Searches were carried out across 12 databases (e.g. MEDLINE, EMBASE). We used the Mixed Methods Appraisal Tool to evaluate study quality, and conducted a narrative synthesis of the data. The review was pre-registered (PROSPERO: CRD42018093453). Thirty-eight studies were identified. Uptake and adherence data were all from trials. Trials recruited healthy participants, those at higher risk of cancer, and those with cancer. Four studies reported moderate to high (40.9-77.7\%) uptake to an aspirin trial among people who were eligible. Most trials (18/22) reported high day-to-day adherence $(\geq 80 \%)$. Three trials observed no association between gender and adherence. One trial found no association between adherence and colorectal cancer risk. Three studies reported moderate to high (43.6-76.0\%) hypothetical willingness to use aspirin. Two studies found that a high proportion of healthcare providers (72.0-76.0\%) perceived aspirin to be a suitable cancer prevention option. No qualitative studies were identified. The likelihood that eligible users of aspirin would participate in a trial evaluating the use of aspirin for preventive therapy was moderate to high. Among participants in a trial, day-to-day adherence was high. Further research is needed to identify uptake and adherence rates in routine care, the factors affecting aspirin use, and the barriers to implementing aspirin into clinical care.
\end{abstract}

\section{Introduction}

Cancer is the second leading cause of death globally (Naghavi et al., 2017), with an estimated 9.6 million cancer deaths worldwide in 2018 (Bray et al., 2018). There is increasing interest in preventive therapy as part of cancer control efforts (Steward and Brown, 2013). A metaanalysis of 45 observational studies found aspirin to be associated with a reduced risk of developing colorectal (relative risk: $0.73,95 \% \mathrm{CI}$ $=0.69-0.78$ ) and other gastrointestinal cancers (range, relative risks: 0.61-0.78) (Bosetti et al., 2020). Reviews have also examined the relationship between aspirin and cancer by synthesising the results of randomised controlled trials (RCTs) investigating aspirin for vascular disease prevention. These results showed that individuals taking aspirin, versus no aspirin, had a reduced 20 -year risk of developing colon cancer (hazard ratio: $0.76,95 \% \mathrm{CI}=0.60-0.96$ ) (Rothwell et al., 2010), and a reduced risk of colorectal cancer death at 10-20 years (hazard ratio: 0.51, 95\% CI = 0.35-0.74) (Rothwell et al., 2011). Cohort studies have observed weaker significant associations between aspirin use and risk reduction of non-gastrointestinal cancers, such as breast (hazard ratio: $0.96,95 \% \mathrm{CI}=0.91-1.00$ ) (Hurwitz et al., 2021), prostate (hazard ratio: 0.95, 95\% CI $=0.90-1.00$ ) (Hurwitz et al., 2021), and lung cancer (relative risk: $0.95,95 \% \mathrm{CI}=0.91-0.98$ ) (Jiang et al., 2021).

Despite many countries having national cancer screening programmes, few have implemented guidance recommending aspirin for cancer prevention. The US Preventive Services Taskforce recommends aspirin for colorectal cancer prevention among adults aged 50-69 who have $\geq 10 \%$ 10-year cardiovascular disease risk (Bibbins-Domingo, 2016). In the UK, the National Institute of Health and Care Excellence

\footnotetext{
* Corresponding author at: Leeds Institute of Health Science, University of Leeds, Clarendon Way, Leeds LS2 9NL, UK.

E-mail address: umkel@leeds.ac.uk (K.E. Lloyd).
} 
recommends daily aspirin for people with Lynch syndrome (National Institute for Health and Clinical Excellence (NICE), 2020), and in Australia aspirin is recommended for the public aged 50-70 (Cancer Council Australia, 2019). Guideline implementation depends on informed uptake, high adherence, and understanding the barriers to achieving these goals. However, deciding whether to use preventive therapy can be a complex choice for patients, and for their healthcare providers prescribing it. The benefits of aspirin need to be considered in relation to its side-effects, as even low doses can increase the risk of gastrointestinal bleeding, ulcers and, in more rare cases, haemorrhagic stroke (Lanas and Scheiman, 2007; Cuzick et al., 2015).

Studies have investigated the barriers and facilitators to using breast cancer preventive therapy. The evidence suggests the factors associated with increased uptake include having children (Hackett et al., 2018), higher objective risk (Smith et al., 2016), higher cancer-related worry (Bober et al., 2004; Holmberg et al., 2017), and fewer concerns about the side-effects (Bober et al., 2004; Thorneloe et al., 2019; Rondanina et al., 2008). Women with lower educational qualifications, depression and those who are older are also less likely to adhere to the medication (Smith et al., 2016). Prospective studies have also identified a positive association between healthcare provider recommendation and patients' use of breast cancer preventive therapy (Bober et al., 2004; Holmberg et al., 2017). To our knowledge, no review has examined decisionmaking in the context of aspirin for cancer prevention among potential users of aspirin and healthcare providers.

We undertook a systematic review to synthesise the quantitative and qualitative data on uptake and adherence behaviours related to aspirin for cancer prevention, investigate the factors affecting decisions to use aspirin, and examine healthcare providers' attitudes towards implementing aspirin in clinical care.

\section{Materials and methods}

\subsection{Search strategy}

We first conducted a search of the literature in March 2018, and reran the searches in February 2020. Searches were conducted in the following databases from inception to February 2020: MEDLINE; EMBASE; CINAHL; Cochrane Library (CENTRAL and Cochrane Database of Systematic Reviews); Database of Abstracts of Reviews of Effects (DARE); NHS Economic Evaluation Database; Pan Health Technology Assessment (HTA) Database; HTA Database (Wiley); PubMed; ProQuest Dissertation and Theses A\&I; and Web of Science Core Collection. We also searched the International Clinical Trials Registry Platform (ICTRP) and Clinical trials.gov, and the websites of Cancer Research UK and canc er.gov for any ongoing trials. After identifying relevant conference abstracts, trials, and dissertations, we searched for the peer-reviewed articles of these studies. Search terms were developed for the concepts: aspirin, cancer and prevention by an information specialist (RR) and project team members using subject headings and free text terms (see supplementary appendix for search strategies). We did not apply date limits or methodological filters to the searches.

We stored and de-duplicated the records in EndNote X9, and screened them using the management software Covidence. To find additional papers, we searched the reference lists of included studies and relevant reviews. The review was pre-registered (PROSPERO number: CRD42018093453), and PRISMA guidelines for reporting were followed throughout (Moher et al., 2009).

\subsection{Study selection}

We included both quantitative and qualitative peer-reviewed studies, which provided empirical data and recruited individuals aged 18 or over. Studies were included if they reported rates of uptake and/or adherence to aspirin (at any dose) for primary or secondary prevention (i.e. preventing recurrence) of cancer. Additionally, we included articles which reported patient, public or healthcare provider attitudes towards using aspirin for cancer prevention. We deviated from the preregistration by including quantitative studies exploring individuals' perceptions about taking aspirin for cancer prevention, instead of only qualitative data. Articles on the same trial were included if they provided additional data, such as adherence at longer follow up. We excluded articles reporting adherence on a smaller sub-sample from an included trial.

As we were only interested in attitudes and behaviour data in the context of aspirin for cancer prevention, we excluded studies where aspirin was not used/prescribed for the primary purpose of cancer prevention. For example, we excluded studies using aspirin for the primary purpose of cardiovascular disease prevention/management, and case control and cohort studies if aspirin was not being used for the primary purpose of cancer prevention. Non-peer reviewed studies and reviews were also excluded. We excluded by hand non-English language studies as we did not have the resources to translate.

Screening of the titles and abstracts was completed by two authors (RJT, KEL), and two authors (RR, LHH) duplicated screening for $20 \%$ of articles. Discrepancies were resolved with a third reviewer (SGS). Two authors (RJT, KEL) screened the full text articles, and second reviewers (LHH, KEL) duplicated screening for $20 \%$ of articles. The review was managed in Covidence.

\subsection{Data extraction}

Two authors (RJT, KEL) extracted the study data using Excel, and $45 \%(17 / 38)$ of a random sample of articles were verified by second reviewers (RR, KEL) to ensure consistency (Shea et al., 2017). We extracted data on study characteristics; sample characteristics; aspirin dose; timing; uptake level; adherence method; adherence definitions; follow-up time; day-to-day adherence; persistence adherence; and factors associated with uptake, day-to-day adherence and/or persistence. Additionally, we extracted data reporting attitudes towards aspirin for cancer prevention.

Uptake rates were defined as the proportion of individuals who were offered aspirin and took the first dose (Vrijens et al., 2012). To calculate uptake to a clinical trial, we calculated the proportion of eligible participants who enrolled on the trial. The denominator was the number of eligible participants offered the trial, with ineligible participants excluded from the calculation. We classified participants who declined trial participation for unknown reasons as declining to take part. We defined day-to-day adherence as the extent to which people took the medication as prescribed (Vrijens et al., 2012). Data could be continuous $(0-100 \%$ of medications) or categorical (proportion classified as adherent). We defined persistence as the length of time between uptake and last dose (Vrijens et al., 2012). Studies reporting the proportion of participants who completed the trial, without explicit reference to the medication, were excluded. We included both self-report and objective adherence measures.

\subsection{Quality assessment}

We used the Mixed Method Appraisal Tool (MMAT) to assess methodological quality (Pluye and Hong, 2014). MMAT is reliable (Pace et al., 2012), and has been used in a review examining decision-making in breast cancer preventive therapy (Smith et al., 2016). For each study design (qualitative, quantitative RCTs, non-randomised quantitative studies, quantitative descriptive studies, mixed methods studies), there was a quality checklist consisting of 5 items. All items were categorised as 'Yes', 'No', or 'Can't tell'.

RCTs received a quality assessment score ranging from 0 to 4 , as the criterion 'Did the participants adhere to the assigned intervention?' (2.5) was removed due to adherence being a review outcome. All other study types received a score $0-5$. The MMAT guidance recommended study teams agreed on an acceptable dropout rate for the criterion 'Are 
there complete outcome data?' $(2.3,3.3)$. We decided a priori that an article would qualify as 'Yes' if they reported a dropout rate of $\leq 30 \%$ participants (Pluye and Hong, 2014; Furlan et al., 2009). One author (KEL) assessed the quality of all articles, with over 35\% (14/38) of a random sample of articles verified by a second author ( $\mathrm{LHH})$ to ensure consistency. Any discrepancies were resolved with a third author (SGS).

\subsection{Synthesis of the evidence}

To determine if a meta-analysis was appropriate we considered whether the included studies were sufficiently similar on the domains of participants (setting), intervention, comparison and outcomes (Ryan and Cochrane Consumers and Communication Review Group, 2016). There was substantial heterogeneity, for example there was high variations in the doses of aspirin prescribed (intervention), assessments of adherence (outcomes), and the participant population (setting). Within subgroups, few studies used the same setting, intervention, and outcome. Therefore, we concluded that a meta-analysis was inappropriate for our review due to the high heterogeneity. Instead we conducted a narrative synthesis, with findings tabulated (Petticrew and Roberts, 2006). We organised the studies into categories and synthesised the findings (Petticrew and Roberts, 2006). Where possible, comparisons were made between studies on the setting (trial vs. routine care), sample population, aspirin dose/frequency, and healthcare provider population. Across the different categories, we also examined if there was a relationship between year of study, and age of the sample, on the review outcomes.

\section{Results}

We identified 17,344 papers, of which 11,258 papers remained after duplicates were removed (Fig. 1). After screening titles and abstracts, we excluded 10,061 articles. We screened 1197 full text articles, 37 studies met the eligibility criteria, and one study was identified by backwards citation searching. A total of 38 studies were included.

\subsection{Uptake of aspirin}

Four studies reported data on uptake of participants to an aspirin clinical trial (Logan et al., 2008; Rexrode et al., 2000; Hull et al., 2018; Jankowski et al., 2018), and all investigated aspirin for primary cancer prevention (Table 1). No studies were identified reporting uptake rates in routine care. All studies were RCTs (Logan et al., 2008; Rexrode et al., 2000; Hull et al., 2018; Jankowski et al., 2018), and of mixed quality with scores ranging from one (Rexrode et al., 2000) to four (Hull et al., 2018) on the MMAT. Three studies (75\%) recruited participants at higher risk of developing cancer (Logan et al., 2008; Hull et al., 2018; Jankowski et al., 2018), and one (25\%) recruited a healthy population sample (Rexrode et al., 2000). The dose and frequency of prescribed aspirin varied, from $100 \mathrm{mg}$ every alternative day (Rexrode et al., 2000) to $325 \mathrm{mg}$ administered daily (Jankowski et al., 2018). Rates of uptake among eligible people to an aspirin trial were moderate to high (40.9-77.7\%) (Logan et al., 2008; Rexrode et al., 2000; Hull et al., 2018; Jankowski et al., 2018).

Rates of uptake to an aspirin trial did not appear to increase or decrease over time. For example, the oldest study conducted in 2000 reported an uptake rate of $61.2 \%$ (Rexrode et al., 2000), while two studies conducted in 2018 reported uptake rates of $40.9 \%$ (Hull et al., 2018) and 77.7\% (Jankowski et al., 2018). A trial with a mean sample age of 65 years observed lower rates of uptake among eligible people (40.9\%) (Hull et al., 2018), compared with studies with a mean sample age of 58 (65.5-77.7\%) (Logan et al., 2008; Jankowski et al., 2018). No studies examined the demographic, psychological or clinical factors associated with uptake. No studies compared different aspirin doses and uptake. See supplementary Table 1 for the proportion of participants who enrolled onto the trial, with the dominator the number of participants offered the trial (i.e. inclusive of ineligible participants).

\subsection{Adherence to aspirin}

A total of 29 studies reported aspirin adherence data (Logan et al., 2008; Hull et al., 2018; Jankowski et al., 2018; Barnes et al., 1999; Burney et al., 1996; Cook et al., 2013; Duggan et al., 2014; Frommel et al., 1997; Krishnan et al., 2001; Lipton et al., 1982; Roop et al., 2013; Roy et al., 2017; Ruffin et al., 1997; Sample et al., 2002a; Sandler et al., 2003; Liesenfeld et al., 2016; Benamouzig et al., 2001; Benamouzig et al., 2003; Benamouzig et al., 2012; Ishikawa et al., 2013; Baron et al., 2003; Burn et al., 2008; Burn et al., 2013; Falk et al., 2012; Pommergaard et al., 2016; Sample et al., 2002b; Garland et al., 2019; Joharatnam-Hogan et al., 2019; Sinicrope et al., 2019), and of these 83\% (24/ 29) were RCTs (Table 2) (Logan et al., 2008; Hull et al., 2018; Jankowski et al., 2018; Cook et al., 2013; Duggan et al., 2014; Lipton et al., 1982; Roop et al., 2013; Roy et al., 2017; Sample et al., 2002a; Sandler et al., 2003; Liesenfeld et al., 2016; Benamouzig et al., 2001; Benamouzig et al., 2003; Benamouzig et al., 2012; Ishikawa et al., 2013; Baron et al., 2003; Burn et al., 2008; Burn et al., 2013; Falk et al., 2012; Pommergaard et al., 2016; Sample et al., 2002b; Garland et al., 2019; Joharatnam-Hogan et al., 2019; Sinicrope et al., 2019). Study quality was mixed according to the MMAT scoring, with 48\% (14/29) of studies assessed as medium (3/4 or $3 / 5)$ or high ( $4 / 5$ or $4 / 4)$ quality (Logan et al., 2008; Hull et al., 2018; Barnes et al., 1999; Cook et al., 2013; Frommel et al., 1997; Krishnan et al., 2001; Roy et al., 2017; Ruffin et al., 1997; Sandler et al., 2003; Ishikawa et al., 2013; Baron et al., 2003; Burn et al., 2013; Pommergaard et al., 2016; Joharatnam-Hogan et al., 2019). The sample characteristics varied, with nearly half of studies $(16 / 29,55 \%)$ recruiting a population at increased risk of developing cancer (Logan et al., 2008; Hull et al., 2018; Jankowski et al., 2018; Barnes et al., 1999; Sample et al., 2002a; Benamouzig et al., 2001; Benamouzig et al., 2003; Benamouzig et al., 2012; Ishikawa et al., 2013; Baron et al., 2003; Burn et al., 2008; Burn et al., 2013; Falk et al., 2012; Pommergaard et al., 2016; Sample et al., 2002b; Garland et al., 2019), such as patients with colorectal adenomas. Five studies (17\%) recruited participants with or who previously had cancer (Frommel et al., 1997; Lipton et al., 1982; Roop et al., 2013; Sandler et al., 2003; JoharatnamHogan et al., 2019), and five (17\%) studies recruited healthy populations (Burney et al., 1996; Cook et al., 2013; Duggan et al., 2014; Ruffin et al., 1997; Liesenfeld et al., 2016). Three studies recruited mixed populations (e.g. higher risk, general public) (Krishnan et al., 2001; Roy et al., 2017; Sinicrope et al., 2019). Most studies investigated aspirin for gastrointestinal cancer prevention, however five studies (17\%) examined the relationship between aspirin and the prevention of non-gastrointestinal cancers. These were lung (Cook et al., 2013; Garland et al., 2019), breast (Cook et al., 2013; Duggan et al., 2014; Roop et al., 2013; Joharatnam-Hogan et al., 2019), and prostate cancer (Joharatnam-Hogan et al., 2019).

There was high heterogeneity across the studies, with multiple definitions of day-to-day adherence, ranging from the proportion who took $\geq 80 \%$ of aspirin (Burney et al., 1996; Krishnan et al., 2001; Roy et al., 2017; Ruffin et al., 1997; Burn et al., 2008; Falk et al., 2012), and percentage of pills taken (Hull et al., 2018; Duggan et al., 2014; Sample et al., 2002a; Benamouzig et al., 2001; Benamouzig et al., 2003; Benamouzig et al., 2012; Pommergaard et al., 2016; Garland et al., 2019). Doses of aspirin were administered from $40.5 \mathrm{mg}$ daily (Ruffin et al., 1997) to $600 \mathrm{mg}$ twice daily (Lipton et al., 1982). Adherence measures varied, with 15 out of 29 studies (52\%) using objective measures (e.g. pill count, Medication Event Monitoring System (MEMS)) (Barnes et al., 1999; Duggan et al., 2014; Frommel et al., 1997; Lipton et al., 1982; Roop et al., 2013; Roy et al., 2017; Liesenfeld et al., 2016; Benamouzig et al., 2001; Benamouzig et al., 2003; Benamouzig et al., 2012; Burn et al., 2008; Burn et al., 2013; Pommergaard et al., 2016; Garland et al., 2019; Sinicrope et al., 2019). Seven studies (24\%) used self-report measures (Hull et al., 2018; Cook et al., 2013; Sandler et al., 2003; 


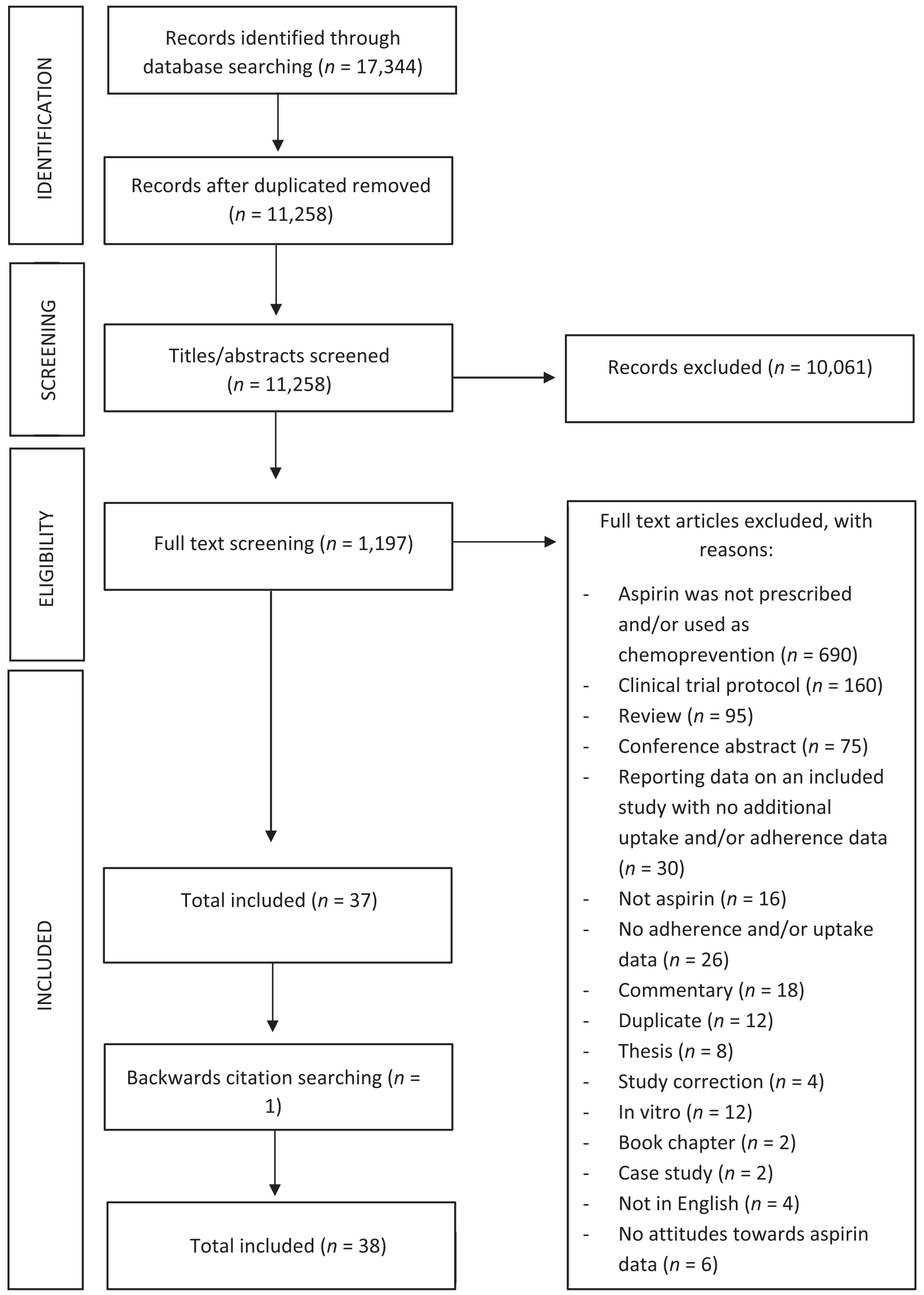

Fig. 1. Flow diagram of search strategy. 
Table 1

Characteristics of articles reporting uptake rates to a clinical trial involving the use of aspirin for cancer prevention $(n=4)$.

\begin{tabular}{|c|c|c|c|c|c|c|c|}
\hline Study & Country & $\begin{array}{l}\text { Design and } \\
\text { quality }\end{array}$ & Population & Dose/timing & $n^{*}$ & Age, years & $\begin{array}{l}\text { Eligible participant } \\
\text { trial uptake** }\end{array}$ \\
\hline $\begin{array}{l}\text { Hull et al., } \\
2018\end{array}$ & UK & $\begin{array}{l}\text { RCT } \\
\text { MMAT } \\
\text { score: } 4\end{array}$ & $\begin{array}{l}\text { Higher risk patients with } \\
\text { colorectal adenomas }\end{array}$ & $\begin{array}{l}300 \mathrm{mg} / \text { daily and/or } \\
\text { eicosapentaenoic acid }\end{array}$ & 709 & Mean: 65 & $40.9 \%$ \\
\hline $\begin{array}{l}\text { Jankowski } \\
\text { et al., } 2018\end{array}$ & $\begin{array}{l}\text { UK and } \\
\text { Canada }\end{array}$ & $\begin{array}{l}\text { RCT } \\
\text { MMAT } \\
\text { score: } 2\end{array}$ & $\begin{array}{l}\text { Patients with Barrett's } \\
\text { oesophagus }\end{array}$ & $\begin{array}{l}300 \mathrm{mg} / \text { daily (UK) or } 325 \mathrm{mg} / \text { daily } \\
\text { (Canada) plus esomeprazole }\end{array}$ & 2557 & Mean: 58 & $77.7 \%$ \\
\hline $\begin{array}{l}\text { Logan et al., } \\
2008\end{array}$ & UK & $\begin{array}{l}\text { RCT } \\
\text { MMAT } \\
\text { score: } 3\end{array}$ & $\begin{array}{l}\text { Higher risk patients with } \\
\text { colorectal adenomas }\end{array}$ & $\begin{array}{l}300 \mathrm{mg} / \text { daily or } 300 \mathrm{mg} \text { plus folate/ } \\
\text { daily }\end{array}$ & 939 & $\begin{array}{l}\text { Mean (range): } 57.8 \\
(27.6-74.6)\end{array}$ & $65.5 \%$ \\
\hline $\begin{array}{l}\text { Rexrode et al., } \\
2000\end{array}$ & US & $\begin{array}{l}\text { RCT } \\
\text { MMAT } \\
\text { score: } 1\end{array}$ & $\begin{array}{l}\text { Women healthcare } \\
\text { providers aged } \geq 45\end{array}$ & $100 \mathrm{mg}$ /alternate day plus vitamin $\mathrm{E}$ & 39,876 & $\begin{array}{l}45-54(60.2 \%) ; 55-64 \\
(29.5 \%) ;>65(10.3 \%)\end{array}$ & $61.2 \%$ \\
\hline
\end{tabular}

Key: RCT $=$ Randomised Control Trial; MMAT = Mixed Methods Appraisal Tool; $n^{*}=$ number of participants enrolled at the beginning of the study; Eligible participant trial uptake $* *$ proportion of eligible individuals who enrolled on the trial, excluding participants who were ineligible.

Ishikawa et al., 2013; Baron et al., 2003; Sample et al., 2002b; Joharatnam-Hogan et al., 2019), and five studies (17\%) used a combination of self-report and objective measures (Logan et al., 2008; Burney et al., 1996; Krishnan et al., 2001; Ruffin et al., 1997; Sample et al., 2002a). Two studies did not report their adherence measurement (Jankowski et al., 2018; Falk et al., 2012).

Day-to-day adherence estimates varied (30.0-100.0\%), however $82 \%$ (18/22 studies) reported high adherence rates of aspirin $(\geq 80.0 \%$ adherence levels) (Hull et al., 2018; Barnes et al., 1999; Duggan et al., 2014; Krishnan et al., 2001; Roy et al., 2017; Ruffin et al., 1997; Sample et al., 2002a; Benamouzig et al., 2001; Benamouzig et al., 2003; Benamouzig et al., 2012; Ishikawa et al., 2013; Baron et al., 2003; Burn et al., 2008; Falk et al., 2012; Pommergaard et al., 2016; Garland et al., 2019; Joharatnam-Hogan et al., 2019; Sinicrope et al., 2019). High levels of day-to-day adherence ( $\geq 80.0 \%$ ) were observed across studies using selfreport measures (Hull et al., 2018; Ishikawa et al., 2013; Baron et al., 2003; Joharatnam-Hogan et al., 2019) and those using objective adherence measures (Barnes et al., 1999; Duggan et al., 2014; Benamouzig et al., 2001; Benamouzig et al., 2003; Benamouzig et al., 2012; Burn et al., 2008; Pommergaard et al., 2016; Garland et al., 2019; Sinicrope et al., 2019). Four studies reported on day-to-day adherence three to four years after participants started aspirin (Logan et al., 2008; Benamouzig et al., 2012; Baron et al., 2003; Pommergaard et al., 2016). Of these studies, three observed high adherence levels $(\geq 80 \%)$ (Benamouzig et al., 2012; Baron et al., 2003; Pommergaard et al., 2016). One RCT reported data on healthy participants for eight years in the active trial, and for 15 years post-trial (Cook et al., 2013). At eight years, 64.0\% of participants were classed as adherent (Cook et al., 2013). By 15 years, $46.0 \%$ were adherent (Cook et al., 2013). No pattern was observed between participants' age and day-to-day adherence.

There was no clear evidence of a relationship between dose and dayto-day adherence. In an RCT of high-risk participants, lower adherence was reported among those taking $650 \mathrm{mg}$ of aspirin (79.0\% adherent), compared with those taking aspirin at $325 \mathrm{mg}$ (100.0\% adherent) and $81 \mathrm{mg}$ (93.0\% adherent) (Sample et al., 2002a). Three other studies reported adherence rates across different doses of aspirin and identified few differences (Benamouzig et al., 2012; Baron et al., 2003; Falk et al., 2012). We also observed no pattern between when the study was conducted (older vs. newer studies) and day-to-day adherence.

Persistence was reported by $52 \%$ (15/29) of studies (Logan et al., 2008; Jankowski et al., 2018; Cook et al., 2013; Frommel et al., 1997; Lipton et al., 1982; Roop et al., 2013; Ruffin et al., 1997; Sample et al., 2002a; Sandler et al., 2003; Liesenfeld et al., 2016; Baron et al., 2003; Burn et al., 2013; Pommergaard et al., 2016; Sample et al., 2002b; Garland et al., 2019). Measurements of persistence varied from average number of months/years participants were taking the medication (Jankowski et al., 2018; Cook et al., 2013; Sandler et al., 2003; Burn et al., 2013), to increase in bleeding time (Frommel et al., 1997). Short- term persistence (i.e. weeks, months) was high (83.3-100.0\%) (Frommel et al., 1997; Ruffin et al., 1997; Sample et al., 2002a; Liesenfeld et al., 2016; Garland et al., 2019). The proportion of participants reporting long-term persistence (i.e. years) varied. Three RCTs, all recruiting participants with colorectal adenomas, examined persistence at three years (Logan et al., 2008; Baron et al., 2003; Pommergaard et al., 2016). One RCT observed high levels of persistence, with $93.6 \%$ of participants still taking at least $50 \%$ of the medication at year three (Baron et al., 2003). In contrast, two trials reported low to moderate levels of persistence, with $38.6 \%$ and $66.8 \%$ of participants completing the three-year medication (Logan et al., 2008; Pommergaard et al., 2016). No pattern was observed between the year the study was conducted and persistence with aspirin. Additionally, no pattern was observed between participants' age and persistence with aspirin. For example, both a trial with a mean sample age of 31 (Liesenfeld et al., 2016), and a trial with a mean age of 66 reported high levels of persistence ( $\geq 90 \%$ ) (Frommel et al., 1997).

Four studies examined factors associated with day-to-day adherence. A non-randomised trial of healthy participants found self-report measures to be significantly associated with higher adherence $(73.0 \%$ adherent), than the objective measure of MEMS (44.0\% adherent) (Burney et al., 1996). Two RCTs and one non-randomised trial observed no association between adherence and gender (Burney et al., 1996; Benamouzig et al., 2001; Benamouzig et al., 2003). In an RCT of participants with history of colorectal adenomas, no association was found between adherence and being at higher risk of recurrence, when compared with those at lower risk (Benamouzig et al., 2001). No other factors associated with day-to-day adherence or persistence were reported.

\subsection{Attitudes towards the use of aspirin for cancer preventive therapy}

\subsubsection{High risk and general public}

Five quantitative descriptive studies examined individuals' attitudes towards using aspirin for the primary prevention of cancer (Yachimski et al., 2015; Jensen et al., 2016; Hur et al., 2009; Hur et al., 2008; Nguyen et al., 2019) (Table 3). All studies were of low (2/5) or medium (3/5) quality, and all were cross-sectional surveys. Three studies (60\%) recruited healthy population samples (Jensen et al., 2016; Hur et al., 2009; Nguyen et al., 2019), and two studies (40\%) recruited patients with Barrett's oesophagus (Yachimski et al., 2015; Hur et al., 2008). Four studies reported moderate to high willingness from participants to use aspirin for cancer prevention (43.6-76.0\%) (Yachimski et al., 2015; Hur et al., 2009; Hur et al., 2008; Nguyen et al., 2019).

Mixed results were observed for an association between participants' demographic characteristics and whether they would use aspirin for cancer prevention. A US survey examined the relationship between healthy participants' characteristics and intentions to use aspirin 
Table 2

Characteristics of articles reporting adherence to aspirin for cancer prevention $(n=29)$.

\begin{tabular}{|c|c|c|c|c|c|c|c|c|c|c|c|c|}
\hline $\begin{array}{l}\text { Study and } \\
\text { location }\end{array}$ & $\begin{array}{l}\text { Design and } \\
\text { quality }\end{array}$ & Population & Dose/timing & $n^{*}$ & Age, years & $\begin{array}{l}\text { Adherence } \\
\text { measure }\end{array}$ & $\begin{array}{l}\text { Day-to-day } \\
\text { adherence } \\
\text { definition }\end{array}$ & $\begin{array}{l}\text { Persistence } \\
\text { adherence } \\
\text { definition }\end{array}$ & $\begin{array}{l}\text { Follow-up } \\
\text { time }\end{array}$ & $\begin{array}{l}\text { Day-to-day } \\
\text { adherence }\end{array}$ & $\begin{array}{l}\text { Persistence } \\
\text { adherence }\end{array}$ & $\begin{array}{l}\text { Associations with } \\
\text { adherence/ } \\
\text { persistence }\end{array}$ \\
\hline $\begin{array}{l}\text { Barnes et al., } \\
1999 \\
\text { US }\end{array}$ & $\begin{array}{l}\text { Non- } \\
\text { randomised } \\
\text { MMAT } \\
\text { score: } 4\end{array}$ & $\begin{array}{l}\text { Adenomatous } \\
\text { polyps }\end{array}$ & $81 \mathrm{mg} /$ daily & 10 & $\begin{array}{l}\text { Mean (range) } \\
53.6(47-64)\end{array}$ & Pill count & $\begin{array}{l}\% \text { who took } \\
\text { medication }\end{array}$ & - & 3 months & $100.0 \%$ & - & None reported \\
\hline $\begin{array}{l}\text { Baron et al., } \\
2003 \\
\text { US and } \\
\text { Canada }\end{array}$ & $\begin{array}{l}\text { RCT } \\
\text { MMAT } \\
\text { score: } 3\end{array}$ & $\begin{array}{l}\text { Colorectal } \\
\text { adenomas }\end{array}$ & $\begin{array}{l}81 \mathrm{mg} / \text { daily or } 325 \mathrm{mg} / \\
\text { daily }\end{array}$ & 1121 & $\begin{array}{l}\text { Mean (SD): } \\
57.3(9.9) \text { - } \\
57.7(9.1)\end{array}$ & Self-report & $\begin{array}{l}\% \text { who took } \\
6-7 \text { tablets/ } \\
\text { week }\end{array}$ & $\begin{array}{l}\% \text { who took } \\
\geq 50 \% \text { tablets in } \\
\text { final year of trial }\end{array}$ & $\begin{array}{l}\text { Approx. } 3 \\
\text { years }\end{array}$ & $\begin{array}{l}81 \mathrm{mg} \\
\text { aspirin: } \\
89.8 \% \\
325 \mathrm{mg} \\
\text { aspirin: } \\
88.0 \% \\
\text { Placebo: } \\
87.1 \%\end{array}$ & $\begin{array}{l}\text { Year 1: } \\
97.8 \% \\
\text { Year 3: } \\
93.6 \%\end{array}$ & None reported \\
\hline $\begin{array}{l}\text { Benamouzig } \\
\text { et al., } 2001 \\
\text { France }\end{array}$ & $\begin{array}{l}\text { RCT } \\
\text { MMAT } \\
\text { score: } 1\end{array}$ & $\begin{array}{l}\text { Colorectal } \\
\text { adenomatous } \\
\text { polyps }\end{array}$ & $\begin{array}{l}300 \mathrm{mg} / \text { daily or } 160 \mathrm{mg} / \\
\text { daily }\end{array}$ & 274 & $\begin{array}{l}\text { Mean (SD) } \\
57.7(9.4)\end{array}$ & Pill count & $\begin{array}{l}\% \text { of pills } \\
\text { taken }\end{array}$ & - & $\begin{array}{l}16 \\
\text { months }\end{array}$ & $84.1 \%$ & - & $\begin{array}{l}\text { No association } \\
\text { with risk (ND) })^{+} \\
\text {No association } \\
\text { with gender } \\
(\mathrm{ND})^{+}\end{array}$ \\
\hline $\begin{array}{l}\text { Benamouzig } \\
\text { et al., } 2003 \\
\text { France }\end{array}$ & $\begin{array}{l}\text { RCT } \\
\text { MMAT } \\
\text { score: } 1\end{array}$ & & & 272 & & & $\begin{array}{l}\text { Mean \% of } \\
\text { pills taken }\end{array}$ & - & $\begin{array}{l}\text { Approx. } 1 \\
\text { year }\end{array}$ & $\begin{array}{l}\text { Aspirin: } \\
\text { 87.0\% } \\
\text { Placebo: } \\
88.0 \%\end{array}$ & - & $\begin{array}{l}\text { No association } \\
\text { with risk (ND) } \\
\text { No association } \\
\text { with gender } \\
(\mathrm{ND})^{+}\end{array}$ \\
\hline $\begin{array}{l}\text { Benamouzig } \\
\text { et al., } 2012 \\
\text { France }\end{array}$ & $\begin{array}{l}\text { RCT } \\
\text { MMAT } \\
\text { score: } 2\end{array}$ & & & & & & $\begin{array}{l}\text { Mean \% of } \\
\text { pills taken }\end{array}$ & - & $\begin{array}{l}\text { Approx. } 4 \\
\text { years }\end{array}$ & $88.0 \%$ & - & $\begin{array}{l}\text { Adherence similar } \\
\text { between aspirin } \\
160 \mathrm{mg} / \text { day vs. } \\
\text { aspirin } 300 \mathrm{mg} / \\
\text { day vs. placebo } \\
(\mathrm{ND})^{+}\end{array}$ \\
\hline $\begin{array}{l}\text { Burn et al., } \\
2008 \\
\text { International }\end{array}$ & $\begin{array}{l}\text { RCT } \\
\text { MMAT } \\
\text { score: } 2\end{array}$ & LS & $\begin{array}{l}300 \mathrm{mg} / \text { twice daily plus } \\
\text { resistant starch }\end{array}$ & 937 & $\begin{array}{l}\text { Mean } \\
\text { (range): } 45 \\
\text { (25-79) }\end{array}$ & Pill count & $\begin{array}{l}\% \text { who took } \\
\text { the tablets } \\
\geq 80.0 \% \text { of } \\
\text { the time }\end{array}$ & - & $\begin{array}{l}\text { Approx. } 2 \\
\text { years }\end{array}$ & $81.0 \%$ & - & None reported \\
\hline $\begin{array}{l}\text { Burn et al., } \\
2013 \\
\text { International }\end{array}$ & $\begin{array}{l}\text { RCT } \\
\text { MMAT } \\
\text { score: } 3\end{array}$ & & & & & & $\begin{array}{l}\% \text { who took } \\
1400 \text { ( } 300 \\
\text { mg) pills } \geq 2 \\
\text { years }\end{array}$ & $\begin{array}{l}\text { Mean duration } \\
\text { of treatment }\end{array}$ & & $\begin{array}{l}\text { Aspirin: } \\
30.0 \% \\
\text { Placebo: } \\
29.1 \%\end{array}$ & $\begin{array}{l}\text { Mean: } 25.2 \\
\text { months }\end{array}$ & None reported \\
\hline $\begin{array}{l}\text { Burney et al., } \\
1996 \\
\text { US }\end{array}$ & $\begin{array}{l}\text { Non- } \\
\text { randomised } \\
\text { MMAT } \\
\text { score: } 2\end{array}$ & Healthy adults & Up to $640 \mathrm{mg} /$ daily & 64 & Not reported & $\begin{array}{l}\text { Self-report } \\
\text { and MEMS }\end{array}$ & $\begin{array}{l}\% \text { who took } \\
\geq 80.0 \% \text { of } \\
\text { the pills }\end{array}$ & - & 14 days & $\begin{array}{l}\text { Self-report: } \\
73.0 \% \\
\text { MEMS: } \\
44.0 \% \\
\text { Self-report } \\
\text { and MEMS: } \\
35.0 \%\end{array}$ & - & $\begin{array}{l}\text { Self-report vs. } \\
\text { MEMS }(p=0.002) \\
\text { No association } \\
\text { with gender }(p= \\
0.95, p=0.78)\end{array}$ \\
\hline $\begin{array}{l}\text { Cook et al., } \\
2013 \\
\text { US }\end{array}$ & $\begin{array}{l}\text { RCT } \\
\text { MMAT } \\
\text { score: } 3\end{array}$ & $\begin{array}{l}\text { Healthy female } \\
\text { healthcare } \\
\text { providers }\end{array}$ & $\begin{array}{l}100 \mathrm{mg} / \text { alternate day } \\
\text { Plus vitamin } \mathrm{E}\end{array}$ & 39,876 & Mean: 55 & Self-report & $\begin{array}{l}\text { Active trial: } \\
\% \text { took } \geq 2 / 3 \\
\text { of aspirin } \\
\text { Post-trial: } \% \\
\text { took aspirin } \\
\geq 3 \text { days per } \\
\text { month }\end{array}$ & $\begin{array}{l}\text { Median } \\
\text { duration of } \\
\text { treatment }\end{array}$ & $\begin{array}{l}\text { Active } \\
\text { trial: } 8 \\
\text { years } \\
\text { Post-trial: } \\
15 \text { years }\end{array}$ & $\begin{array}{l}\text { Active trial: } \\
\text { Aspirin } \\
(64.0 \%) \\
\text { Placebo } \\
(65.0 \%) \\
\text { Post-trial: } \\
\text { Aspirin } \\
(46.0 \%)\end{array}$ & $\begin{array}{l}\text { Median: } 9 \\
\text { years }\end{array}$ & None reported \\
\hline
\end{tabular}




\begin{tabular}{|c|c|c|c|c|c|c|c|c|c|c|c|c|}
\hline $\begin{array}{l}\text { Study and } \\
\text { location }\end{array}$ & $\begin{array}{l}\text { Design and } \\
\text { quality }\end{array}$ & Population & Dose/timing & $n^{*}$ & Age, years & $\begin{array}{l}\text { Adherence } \\
\text { measure }\end{array}$ & $\begin{array}{l}\text { Day-to-day } \\
\text { adherence } \\
\text { definition }\end{array}$ & $\begin{array}{l}\text { Persistence } \\
\text { adherence } \\
\text { definition }\end{array}$ & $\begin{array}{l}\text { Follow-up } \\
\text { time }\end{array}$ & $\begin{array}{l}\text { Day-to-day } \\
\text { adherence }\end{array}$ & $\begin{array}{l}\text { Persistence } \\
\text { adherence }\end{array}$ & $\begin{array}{l}\text { Associations with } \\
\text { adherence/ } \\
\text { persistence }\end{array}$ \\
\hline & & & & & & & & & & $\begin{array}{l}\text { placebo } \\
(43.0 \%)\end{array}$ & & \\
\hline $\begin{array}{l}\text { Duggan et al., } \\
2014 \\
\text { US }\end{array}$ & $\begin{array}{l}\text { RCT } \\
\text { MMAT } \\
\text { score: } 2\end{array}$ & $\begin{array}{l}\text { Post- } \\
\text { menopausal } \\
\text { women }\end{array}$ & $325 \mathrm{mg} /$ daily & 144 & $\begin{array}{l}\text { Mean (SD): } \\
59.4 \text { (5.4) }\end{array}$ & Pill count & $\begin{array}{l}\% \text { of pills } \\
\text { taken }\end{array}$ & - & 6 months & $\begin{array}{l}\text { Aspirin } \\
(87.0 \%) \\
\text { placebo } \\
(87.0 \%)\end{array}$ & - & None reported \\
\hline $\begin{array}{l}\text { Falk et al., } 2012 \\
\text { US, Canada, } \\
\text { Puerto Rico }\end{array}$ & $\begin{array}{l}\text { RCT } \\
\text { MMAT } \\
\text { score: } 2\end{array}$ & $\begin{array}{l}\text { Barrett's } \\
\text { Oesophagus }\end{array}$ & $\begin{array}{l}81 \mathrm{mg} / \text { daily or } 325 \mathrm{mg} / \\
\text { daily } \\
\text { Plus esomeprazole }\end{array}$ & 122 & $\begin{array}{l}\text { Mean (SD): } \\
59.7 \text { (11.2) }\end{array}$ & Not reported & $\begin{array}{l}\text { Median } \\
\text { number of } \\
\text { tablets taken } \\
\text { Percentage of } \\
\text { adherence } \\
\text { (median) }\end{array}$ & - & 28 days & $\begin{array}{l}27-28 \\
\text { tablets for } \\
\text { aspirin and } \\
\text { placebo } \\
\text { (median) } \\
100.0 \% \\
\text { (median) }\end{array}$ & - & None reported \\
\hline $\begin{array}{l}\text { Frommel et al., } \\
1997 \\
\text { US }\end{array}$ & $\begin{array}{l}\text { Non- } \\
\text { randomised } \\
\text { MMAT } \\
\text { score: } 3\end{array}$ & CRC & $\begin{array}{l}325 \mathrm{mg} / \text { daily then } 325 \\
\mathrm{mg} / \text { twice daily }\end{array}$ & 17 & $\begin{array}{l}\text { Mean (SD): } \\
65.6(13.6)\end{array}$ & Bleeding time & - & $\begin{array}{l}\text { Increase in } \\
\text { bleeding time at } \\
120 \text { days }\end{array}$ & 120 days & - & $94.1 \%$ & None reported \\
\hline $\begin{array}{l}\text { Garland et al., } \\
2019 \\
\text { US }\end{array}$ & $\begin{array}{l}\text { RCT } \\
\text { MMAT } \\
\text { score: } 2\end{array}$ & $\begin{array}{l}\text { High risk of lung } \\
\text { cancer }\end{array}$ & $\begin{array}{l}\text { Intermittent: } 81 \mathrm{mg} / \text { daily } \\
\text { one week/placebo one } \\
\text { week } \\
\text { Continuous: } 81 \mathrm{mg} / \text { daily }\end{array}$ & 54 & $\begin{array}{l}\text { Mean (SD): } \\
52(8)\end{array}$ & Pill count & $\begin{array}{l}\text { Mean \% of } \\
\text { pills taken }\end{array}$ & $\begin{array}{l}\% \text { who } \\
\text { completed the } \\
\text { intervention }\end{array}$ & 12 weeks & $98.0 \%$ & $83.3 \%$ & None reported \\
\hline $\begin{array}{l}\text { Hull et al., } 2018 \\
\text { UK }\end{array}$ & $\begin{array}{l}\text { RCT } \\
\text { MMAT } \\
\text { score: } 4\end{array}$ & $\begin{array}{l}\text { Colorectal } \\
\text { adenomas }\end{array}$ & $\begin{array}{l}300 \mathrm{mg} / \text { daily and/or } \\
\text { eicosapentaenoic acid }\end{array}$ & 709 & Mean: 65 & Self-report & $\begin{array}{l}\text { Mean } \% \text { of } \\
\text { pills taken }\end{array}$ & - & 1 year & $\begin{array}{l}\text { Aspirin: } \\
97.0 \% \\
\text { Placebo: } \\
97.0 \%\end{array}$ & - & None reported \\
\hline $\begin{array}{l}\text { Ishikawa et al., } \\
2013 \\
\text { Japan }\end{array}$ & $\begin{array}{l}\text { RCT } \\
\text { MMAT } \\
\text { score: } 3\end{array}$ & FAP & $100 \mathrm{mg} /$ daily & 34 & $\begin{array}{l}\text { Mean (SD): } \\
36.7(13.9)- \\
39.7(12.8)\end{array}$ & Self-report & Not reported & - & $\begin{array}{l}10 \\
\text { months }\end{array}$ & $\begin{array}{l}\text { Aspirin: } \\
83.3 \% \\
\text { Placebo: } \\
88.4 \%\end{array}$ & - & None reported \\
\hline $\begin{array}{l}\text { Jankowski } \\
\text { et al., } 2018 \\
\text { UK and } \\
\text { Canada }\end{array}$ & $\begin{array}{l}\text { RCT } \\
\text { MMAT } \\
\text { score: } 2\end{array}$ & $\begin{array}{l}\text { Barrett's } \\
\text { oesophagus }\end{array}$ & $\begin{array}{l}300 \mathrm{mg} / \text { daily (UK) or } 325 \\
\mathrm{mg} / \text { daily (Canada) } \\
\text { Plus esomeprazole }\end{array}$ & 2557 & Mean: 58-59 & Not reported & - & $\begin{array}{l}\% \text { still taking } \\
\text { aspirin at } 10 \\
\text { years } \\
\text { Median } \\
\text { duration of } \\
\text { treatment }\end{array}$ & $\begin{array}{l}\text { Approx. } \\
10 \text { years }\end{array}$ & - & $\begin{array}{l}>25 \% \text { still } \\
\text { taking } \\
\text { aspirin at } 10 \\
\text { years } \\
\text { Median: } 8.9 \\
\text { years }\end{array}$ & None reported \\
\hline $\begin{array}{l}\text { Joharatnam- } \\
\text { Hogan et al., } \\
2019 \\
\text { UK }\end{array}$ & $\begin{array}{l}\text { RCT } \\
\text { MMAT } \\
\text { score: } 3\end{array}$ & $\begin{array}{l}\text { Gastro- } \\
\text { oesophageal, } \\
\text { CRC, breast, } \\
\text { prostate cancer }\end{array}$ & $\begin{array}{l}100 \mathrm{mg} / \text { daily or } 300 \mathrm{mg} \text { / } \\
\text { daily }\end{array}$ & 2719 & $\begin{array}{l}\text { Median: } \\
\text { 52-71 }\end{array}$ & Self-report & $\begin{array}{l}\% \text { who took } \\
6-7 \text { tablets/ } \\
\text { week }\end{array}$ & - & 8 weeks & $95.0 \%$ & - & None reported \\
\hline $\begin{array}{l}\text { Krishnan et al., } \\
2001 \\
\text { US }\end{array}$ & $\begin{array}{l}\text { Non- } \\
\text { randomised } \\
\text { MMAT } \\
\text { score: } 4\end{array}$ & $\begin{array}{l}\text { High vs. normal } \\
\text { risk for CRC }\end{array}$ & $81 \mathrm{mg} /$ daily & 92 & $\begin{array}{l}\text { Mean (SD): } \\
36.5(14.8)- \\
55.2(13.9)\end{array}$ & $\begin{array}{l}\text { Self-report } \\
\text { and pill } \\
\text { counts }\end{array}$ & $\begin{array}{l}\% \text { who took } \\
\geq 80.0 \% \text { of } \\
\text { the pills }\end{array}$ & - & 28 days & $100.0 \%$ & - & None reported \\
\hline $\begin{array}{l}\text { Liesenfeld et al., } \\
2016 \\
\text { US }\end{array}$ & $\begin{array}{l}\text { RCT } \\
\text { MMAT } \\
\text { score: } 2\end{array}$ & $\begin{array}{l}\text { Healthy men } \\
\text { and women }\end{array}$ & $325 \mathrm{mg} /$ daily & 40 & $\begin{array}{l}\text { Mean (SD): } \\
31(6.2)\end{array}$ & $\begin{array}{l}\text { Salicylic acid } \\
\text { metabolites }\end{array}$ & - & $\begin{array}{l}\% \text { with salicylic } \\
\text { acid metabolites } \\
\text { detected at } \\
\text { study end }\end{array}$ & 60 days & - & $92.5 \%$ & None reported \\
\hline \multirow[t]{2}{*}{$\begin{array}{l}\text { Lipton et al., } \\
1982 \\
\text { US }\end{array}$} & $\begin{array}{l}\text { RCT } \\
\text { MMAT } \\
\text { score: } 1\end{array}$ & $\begin{array}{l}\text { Dukes } \mathrm{B}_{2} \text { and } \\
\mathrm{CRC} / \text { rectal } \\
\text { cancer }\end{array}$ & $600 \mathrm{mg} / \mathrm{twice}$ daily & 66 & $\begin{array}{l}\text { Not } \\
\text { described }\end{array}$ & $\begin{array}{l}\text { Blood } \\
\text { salicylate } \\
\text { levels }\end{array}$ & - & $\begin{array}{l}\% \text { who had a } \\
\text { salicylate level } \\
\text { of } \geq 4 \mathrm{mg} / \mathrm{dl} \text { at } \\
\text { study end }\end{array}$ & $\begin{array}{l}\text { Not } \\
\text { described }\end{array}$ & - & $83.3 \%$ & None reported \\
\hline & & $\begin{array}{l}\text { Colorectal } \\
\text { adenomas }\end{array}$ & $\begin{array}{l}300 \mathrm{mg} / \text { daily or } 300 \mathrm{mg} \\
\text { plus folate/daily }\end{array}$ & 939 & & $\begin{array}{l}\text { Self-report } \\
\text { and pill count }\end{array}$ & & & $\begin{array}{l}\text { Approx. } 3 \\
\text { years }\end{array}$ & & $66.8 \%$ & None reported \\
\hline
\end{tabular}




\begin{tabular}{|c|c|c|c|c|c|c|c|c|c|c|c|c|}
\hline $\begin{array}{l}\text { Study and } \\
\text { location }\end{array}$ & $\begin{array}{l}\text { Design and } \\
\text { quality }\end{array}$ & Population & Dose/timing & $n^{*}$ & Age, years & $\begin{array}{l}\text { Adherence } \\
\text { measure }\end{array}$ & $\begin{array}{l}\text { Day-to-day } \\
\text { adherence } \\
\text { definition }\end{array}$ & $\begin{array}{l}\text { Persistence } \\
\text { adherence } \\
\text { definition }\end{array}$ & $\begin{array}{l}\text { Follow-up } \\
\text { time }\end{array}$ & $\begin{array}{l}\text { Day-to-day } \\
\text { adherence }\end{array}$ & $\begin{array}{l}\text { Persistence } \\
\text { adherence }\end{array}$ & $\begin{array}{l}\text { Associations with } \\
\text { adherence/ } \\
\text { persistence }\end{array}$ \\
\hline $\begin{array}{l}\text { Logan et al., } \\
2008 \\
\text { UK }\end{array}$ & $\begin{array}{l}\text { RCT } \\
\text { MMAT } \\
\text { score: } 3\end{array}$ & & & & $\begin{array}{l}\text { Mean } \\
\text { (range): } 57.8 \\
(27.6-74.6)\end{array}$ & & $\begin{array}{l}\% \text { who took } \\
\geq 95.0 \% \text { of } \\
\text { the pills }\end{array}$ & $\begin{array}{l}\% \text { who } \\
\text { completed trial } \\
\text { medication }\end{array}$ & & $\begin{array}{l}\text { Aspirin: } \\
75.4 \% \\
\text { Placebo: } \\
76.4 \%\end{array}$ & & \\
\hline $\begin{array}{l}\text { Pommergaard } \\
\text { et al., } 2016 \\
\text { International }\end{array}$ & $\begin{array}{l}\text { RCT } \\
\text { MMAT } \\
\text { score: } 3\end{array}$ & $\begin{array}{l}\text { Colorectal } \\
\text { adenomas }\end{array}$ & $\begin{array}{l}37.5 \mathrm{mg} \text { aspirin with } \\
\text { calcium carbonate/twice } \\
\text { daily } \\
\text { Plus calcitriol }\end{array}$ & 1107 & $\begin{array}{l}\text { Median (SD): } \\
59(8.1)-60 \\
(8.3)\end{array}$ & Pill count & $\begin{array}{l}\text { Median \% of } \\
\text { pills taken }\end{array}$ & $\begin{array}{l}\% \text { who } \\
\text { completed } 3 \\
\text { years of } \\
\text { treatment }\end{array}$ & 3 years & $\begin{array}{l}\text { Aspirin: } \\
99.0 \% \\
\text { Placebo: } \\
99.0 \%\end{array}$ & $38.6 \%$ & None reported \\
\hline $\begin{array}{l}\text { Roop et al., } \\
2013 \\
\text { US }\end{array}$ & $\begin{array}{l}\text { RCT } \\
\text { MMAT } \\
\text { score: } 2\end{array}$ & $\begin{array}{l}\text { Metastatic } \\
\text { breast cancer }\end{array}$ & $\begin{array}{l}325 \mathrm{mg} / \text { daily plus } \\
\text { clopidogrel }\end{array}$ & 48 & $\begin{array}{l}\text { Mean: } \\
50.7-58.4\end{array}$ & $\begin{array}{l}\text { Platelet- } \\
\text { function tests }\end{array}$ & - & $\begin{array}{l}\text { Inhibition of } \\
\text { platelet- } \\
\text { function }\end{array}$ & 4 weeks & - & $p<0.001$ & None reported \\
\hline $\begin{array}{l}\text { Roy et al., } 2017 \\
\text { US }\end{array}$ & $\begin{array}{l}\text { RCT } \\
\text { MMAT } \\
\text { score: } 3\end{array}$ & $\begin{array}{l}\text { Colonoscopy } \\
\text { For adenoma or } \\
\text { CRC resected }\end{array}$ & $325 \mathrm{mg} /$ daily & 79 & $\begin{array}{l}\text { Mean (SD): } \\
54(11)-57 \\
\text { (9) }\end{array}$ & $\begin{array}{l}\text { Clinical } \\
\text { assessment } \\
\text { and pill } \\
\text { counts }\end{array}$ & $\begin{array}{l}\% \text { who took } \\
\geq 80.0 \% \text { of } \\
\text { the pills }\end{array}$ & - & 3-months & $\begin{array}{l}\text { Aspirin: } \\
100.0 \% \\
\text { Placebo: } \\
100.0 \%\end{array}$ & - & None reported \\
\hline $\begin{array}{l}\text { Ruffin et al., } \\
1997 \\
\text { US }\end{array}$ & $\begin{array}{l}\text { Non- } \\
\text { randomised } \\
\text { MMAT } \\
\text { score: } 3\end{array}$ & $\begin{array}{l}\text { Healthy } \\
\text { participants }\end{array}$ & $\begin{array}{l}40.5 \mathrm{mg}, 81 \mathrm{mg}, 162 \mathrm{mg} \text {, } \\
324 \mathrm{mg} \text {, or } 648 \mathrm{mg} / \text { daily }\end{array}$ & 66 & $\begin{array}{l}\text { Mean (range) } \\
27.8 \text { (19-56) }\end{array}$ & $\begin{array}{l}\text { Self-report } \\
\text { and MEMS }\end{array}$ & $\begin{array}{l}\% \text { who took } \\
\text { an extra dose } \\
\text { on day } 15\end{array}$ & $\begin{array}{l}\% \text { who } \\
\text { completed the } \\
\text { protocol }\end{array}$ & 14 days & $\begin{array}{l}40.5 \mathrm{mg}= \\
20.0 \% \\
81 \mathrm{mg}= \\
10.0 \% \\
162 \mathrm{mg}= \\
20.0 \% \\
324 \mathrm{mg}= \\
10.0 \%\end{array}$ & $98.5 \%$ & None reported \\
\hline $\begin{array}{l}\text { Sample et al., } \\
\text { 2002a } \\
\text { US }\end{array}$ & $\begin{array}{l}\text { RCT } \\
\text { MMAT } \\
\text { score: } 1\end{array}$ & $\begin{array}{l}\text { Colorectal } \\
\text { adenomas }\end{array}$ & $\begin{array}{l}81 \mathrm{mg} / \text { daily or } 325 \mathrm{mg} / \\
\text { daily or } 650 \mathrm{mg} / \text { daily }\end{array}$ & 60 & Mean: 58.2 & $\begin{array}{l}\text { Self-report, } \\
\text { pill count; } \\
\text { plasma } \\
\text { salicylate } \\
\text { levels }\end{array}$ & $\begin{array}{l}\% \text { of pills } \\
\text { taken }\end{array}$ & $\begin{array}{l}\text { \% whose plasma } \\
\text { salicylate levels } \\
\text { significantly } \\
\text { exceeded } \\
\text { baseline }\end{array}$ & 4 weeks & $99.0 \%$ & $\begin{array}{l}93.0 \%(81 \\
\mathrm{mg}) ; 100.0 \% \\
(325 \mathrm{mg}) \\
79.0 \%(650 \\
\mathrm{mg})\end{array}$ & None reported \\
\hline $\begin{array}{l}\text { Sample et al., } \\
\text { 2002b } \\
\text { US }\end{array}$ & $\begin{array}{l}\text { RCT } \\
\text { MMAT } \\
\text { score: } 1\end{array}$ & & & 43 & $\begin{array}{l}40-50 \\
(10.5 \%) ; \\
51-60 \\
(36.8 \%) \\
61-70 \\
(52.6 \%)\end{array}$ & Self-report & - & $\begin{array}{l}\% \text { taking aspirin } \\
\text { regularly at } \\
\text { mean } 17.3 \\
\text { months }\end{array}$ & - & - & $41.9 \%$ & None reported \\
\hline $\begin{array}{l}\text { Sandler et al., } \\
2003 \\
\text { US }\end{array}$ & $\begin{array}{l}\text { RCT } \\
\text { MMAT } \\
\text { score: } 3\end{array}$ & CRC & $325 \mathrm{mg} /$ daily & 635 & $\begin{array}{l}\leq 39(1 \%) ; \\
40-49 \\
(14 \%) ; \\
50-59 \\
(24 \%) ; \\
60-69 \\
(33 \%) ; \geq 70 \\
(28 \%)\end{array}$ & Self-report & $\begin{array}{l}\% \text { taking } 7 \\
\text { pills per week }\end{array}$ & $\begin{array}{l}\text { Median } \\
\text { duration of } \\
\text { treatment }\end{array}$ & $\begin{array}{l}\text { Not } \\
\text { reported }\end{array}$ & - & $\begin{array}{l}\text { Median: } \\
30.9 \text { months }\end{array}$ & None reported \\
\hline $\begin{array}{l}\text { Sinicrope et al., } \\
2019 \\
\text { US }\end{array}$ & $\begin{array}{l}\text { RCT } \\
\text { MMAT } \\
\text { score: } 2\end{array}$ & $\begin{array}{l}\text { Advanced } \\
\text { adenomas or } \\
\text { cancer }\end{array}$ & $\begin{array}{l}325 \mathrm{mg} / \text { daily plus } \\
\text { Difluoromethylornithine }\end{array}$ & 104 & $\begin{array}{l}\text { Mean (SD): } \\
62.6(9.09)\end{array}$ & Pill count & $\begin{array}{l}\% \text { who took } \\
\geq 80.0 \% \text { of } \\
\text { the pills }\end{array}$ & - & 1 year & $98.1 \%$ & - & None reported \\
\hline
\end{tabular}

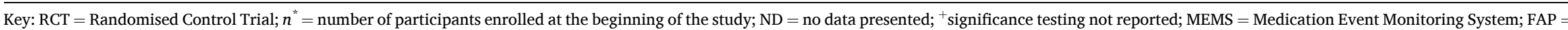
Familial Adenomatous Polyposis; LS = Lynch Syndrome; CRC = Colorectal Cancer. 
Table 3

Characteristics of articles reporting public, patient and healthcare provider attitudes towards using or recommending aspirin for cancer prevention $(n=8)$.

\begin{tabular}{|c|c|c|c|c|c|c|c|c|}
\hline $\begin{array}{l}\text { Study and } \\
\text { location }\end{array}$ & $\begin{array}{l}\text { Design } \\
\text { and } \\
\text { quality }\end{array}$ & Population & Setting & Outcomes & $n^{*}$ & Age, years & $\begin{array}{l}\text { Attitudes towards } \\
\text { aspirin for cancer } \\
\text { prevention }\end{array}$ & $\begin{array}{l}\text { Associations with higher } \\
\text { attitudes (e.g. willingness, } \\
\text { intentions) }\end{array}$ \\
\hline $\begin{array}{l}\text { Chen et al., } \\
2017 \\
\text { Australia }\end{array}$ & $\begin{array}{l}\text { Cross- } \\
\text { sectional } \\
\text { survey } \\
\text { MMAT } \\
\text { score: } 3\end{array}$ & $\begin{array}{l}\text { Clinicians (genetics } \\
\text { providers; } \\
\text { gastroenterologists; } \\
\text { colorectal surgeons) }\end{array}$ & $\begin{array}{l}\text { HCP } \\
\text { survey }\end{array}$ & $\begin{array}{l}\text { - discuss aspirin for } \\
\text { cancer prevention with } \\
\text { patients } \\
\text { - recommends/ } \\
\text { prescribes aspirin to } \\
\text { patients }\end{array}$ & 181 & $\begin{array}{l}<50 \\
(60.0 \%) \\
\geq 50 \\
(40.0 \%)\end{array}$ & $\begin{array}{l}76.0 \% \text { thought } \\
\text { aspirin was } \\
\text { 'somewhat' or } \\
\text { 'very' effective }\end{array}$ & $\begin{array}{l}\text { Univariable analysis: } \\
\text { - professional group } \\
\text { Multivariable analysis: } \\
\text { - no association }\end{array}$ \\
\hline $\begin{array}{l}\text { Das et al., } \\
2008 \\
\text { UK }\end{array}$ & $\begin{array}{l}\text { Cross- } \\
\text { sectional } \\
\text { survey } \\
\text { MMAT } \\
\text { score: } 3\end{array}$ & Gastroenterologists & $\begin{array}{l}\text { HCP } \\
\text { survey }\end{array}$ & $\begin{array}{l}\text { Variation in practice of } \\
\text { BO management }\end{array}$ & 226 & ND & $\begin{array}{l}72.0 \% \text { thought } \\
\text { using aspirin or } \\
\text { COX- } 2 \text { was a good } \\
\text { option }\end{array}$ & None reported \\
\hline $\begin{array}{l}\text { Hur et al., } \\
2008 \\
\text { US }\end{array}$ & $\begin{array}{l}\text { Cross- } \\
\text { sectional } \\
\text { survey } \\
\text { MMAT } \\
\text { score: } 2\end{array}$ & BO patients & $\begin{array}{l}\text { Patient } \\
\text { survey }\end{array}$ & $\begin{array}{l}\text { Patient preferences for } \\
\text { celecoxib and aspirin for } \\
\text { cancer prevention }\end{array}$ & 100 & $\begin{array}{l}\text { Mean } \\
\text { (SD): } 64.5 \\
(11.3)\end{array}$ & $\begin{array}{l}76.0 \% \text { willing to } \\
\text { use aspirin }\end{array}$ & $\begin{array}{l}\text { Univariable analysis: } \\
\text { - younger age } \\
\text { - more educational } \\
\text { qualifications } \\
\text { Multivariable analysis: } \\
\text { - no association }\end{array}$ \\
\hline $\begin{array}{l}\text { Hur et al., } \\
2009 \\
\text { US }\end{array}$ & $\begin{array}{l}\text { Cross- } \\
\text { sectional } \\
\text { survey } \\
\text { MMAT } \\
\text { score: } 2\end{array}$ & Healthy population & $\begin{array}{l}\text { Public } \\
\text { survey }\end{array}$ & $\begin{array}{l}\text { Patient preferences for } \\
\text { celecoxib and aspirin for } \\
\text { cancer prevention }\end{array}$ & 202 & $\begin{array}{l}\text { Median } \\
\text { age group: } \\
\text { 45-54 }\end{array}$ & $\begin{array}{l}43.6 \% \text { willing to } \\
\text { use aspirin }\end{array}$ & $\begin{array}{l}\text { Males }(58.1 \%) \text { more willing to } \\
\text { take aspirin than females } \\
(31.2 \%)\end{array}$ \\
\hline $\begin{array}{l}\text { Jensen } \\
\text { et al., } \\
2016 \\
\text { US }\end{array}$ & $\begin{array}{l}\text { Cross- } \\
\text { sectional } \\
\text { survey } \\
\text { MMAT } \\
\text { score: } 3\end{array}$ & $\begin{array}{l}\text { Healthy population (aged } \\
40-65 \text { ) }\end{array}$ & $\begin{array}{l}\text { Public } \\
\text { survey }\end{array}$ & $\begin{array}{l}\text { Intentions to use aspirin } \\
\text { for cancer prevention on } \\
5 \text {-point scale from } \\
\text { strongly disagree (1) to } \\
\text { strongly agree (5) }\end{array}$ & 1000 & $\begin{array}{l}\text { Mean } \\
\text { (SD): } \\
56.65 \\
(6.87)\end{array}$ & $\begin{array}{l}\text { Intentions to use } \\
\text { aspirin for cancer } \\
\text { prevention }(\mathrm{M}= \\
3.34, \mathrm{SD}=1.22)\end{array}$ & $\begin{array}{l}\text { Demographic variables: } \\
\text { - older } \\
\text { - male } \\
\text { - black ethnicity } \\
\text { Clinical factors: } \\
\text { - did not already take aspirin } \\
\text { - history of polyps } \\
\text { - smoked > } 100 \text { cigarettes } \\
\text { Psychosocial variables: } \\
\text { - increased perceived } \\
\text { susceptibility, barriers, response } \\
\text { and self-efficacy } \\
\text { - reporting less Cancer } \\
\text { information overload }\end{array}$ \\
\hline $\begin{array}{l}\text { Nguyen } \\
\text { et al., } \\
2019 \\
\text { Australia }\end{array}$ & $\begin{array}{l}\text { Cross- } \\
\text { sectional } \\
\text { survey } \\
\text { MMAT } \\
\text { score: } 3\end{array}$ & $\begin{array}{l}\text { Healthy population (aged } \\
50-70 \text { ) }\end{array}$ & $\begin{array}{l}\text { Public } \\
\text { survey }\end{array}$ & $\begin{array}{l}\text { Whether they would } \\
\text { take aspirin for bowel } \\
\text { cancer prevention }\end{array}$ & 304 & $\begin{array}{l}50-54 \\
(24.7 \%) \\
55-59 \\
(29.6 \%) \\
60-64 \\
(21.1 \%) \\
65-70 \\
(24.7 \%)\end{array}$ & $\begin{array}{l}>70.0 \% \text { would } \\
\text { take aspirin }\end{array}$ & $\begin{array}{l}\text { Current aspirin use }(p<0.001) \\
\text { No differences across } \\
\text { demographic factors (gender, } \\
\text { age, education, martial status), } \\
\text { or other clinical factors (family } \\
\text { history of CRC) }\end{array}$ \\
\hline $\begin{array}{l}\text { Smith et al., } \\
2017 \\
\text { UK }\end{array}$ & $\begin{array}{l}\text { Cross- } \\
\text { sectional } \\
\text { survey } \\
\text { MMAT } \\
\text { score: } 3\end{array}$ & GPs practising in the UK & $\begin{array}{l}\text { HCP } \\
\text { survey }\end{array}$ & $\begin{array}{l}\text { Willingness to prescribe } \\
\text { LS patients aspirin at } \\
600 \mathrm{mg}\end{array}$ & 1007 & $\begin{array}{l}<50 \\
(72.3 \%) \\
\geq 50 \\
(27.7 \%)\end{array}$ & $\begin{array}{l}62.3 \% \text { willing to } \\
\text { prescribe aspirin at } \\
600 \mathrm{mg}\end{array}$ & $\begin{array}{l}\text { - } \geq 50 \text { years old } \\
\text { - >10 years' experience } \\
\text { - without special interest in } \\
\text { family history } \\
\text { - greater awareness of } \\
\text { preventive effects aspirin } \\
\text { - having seen LS patient in } \\
\text { practice }\end{array}$ \\
\hline $\begin{array}{l}\text { Yachimski } \\
\text { et al., } \\
2015 \\
\text { US }\end{array}$ & $\begin{array}{l}\text { Cross- } \\
\text { sectional } \\
\text { survey } \\
\text { MMAT } \\
\text { score: } 2\end{array}$ & BO patients & $\begin{array}{l}\text { Patient } \\
\text { survey }\end{array}$ & $\begin{array}{l}\text { Willingness to undergo } \\
\text { treatment A (ablation) } \\
\text { and/or treatment B } \\
\text { (aspirin) }\end{array}$ & 81 & $\begin{array}{l}\text { Mean: } \\
60.2\end{array}$ & $\begin{array}{l}53.0 \% \text { willing to } \\
\text { use aspirin (with } \\
\text { endoscopic } \\
\text { surveillance every } \\
3-5 \text { years) }\end{array}$ & $\begin{array}{l}\text { No differences across } \\
\text { demographic factors (gender, } \\
\text { age, education, ethnicity) and } \\
\text { clinical variables (already } \\
\text { taking aspirin, using PPI, } \\
\text { personal history of cancer, heart } \\
\text { condition, and peptic ulcer) }\end{array}$ \\
\hline
\end{tabular}

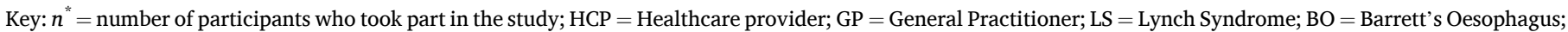
$\mathrm{PPI}=$ Proton Pump Inhibitor; CRC = Colorectal Cancer; ND = No Data.

(Jensen et al., 2016). Higher intentions were significantly associated with being male, black ethnicity, older age, history of polyps, and being a smoker (Jensen et al., 2016). Another survey recruiting Barrett's oesophagus patients found higher education and younger age to be significantly associated with higher willingness to use aspirin in the univariable analysis (Hur et al., 2008). However, this association was not significant in the multivariable analysis (Hur et al., 2008). Two studies also found no evidence of a relationship between demographic factors and willingness to use aspirin (Yachimski et al., 2015; Nguyen et al., 2019). Mixed evidence was also observed for the relationship between participants' current aspirin use and whether they would use aspirin for cancer prevention (Jensen et al., 2016; Nguyen et al., 2019).

Participants with increased self-efficacy, response efficacy, barriers and perceived susceptibility to developing colorectal cancer were significantly more likely to report higher intentions to use aspirin (Jensen et al., 2016). Some of the barriers found to be significantly and positively associated with intentions included participants' believing their doctor would want them to take aspirin, and believing most people 
their age were being told to take aspirin (Jensen et al., 2016). Participants who believed there was low evidence for using aspirin for cancer prevention reported significantly lower intentions (Jensen et al., 2016).

No clear relationship was observed between year of study and attitudes towards aspirin. Two papers examined publics' willingness to use aspirin, with the 2019 Australian study finding higher willingness (>70\%) (Nguyen et al., 2019), than a US-based study conducted in 2009 (43.6\%) (Hur et al., 2009). However, among two US studies, one conducted in 2008 found higher willingness among patients with Barrett's oesophagus (76.0\%) (Hur et al., 2008), compared with a 2015 study examining willingness among the same patient population (53.0\%) (Yachimski et al., 2015).

\subsubsection{Healthcare providers}

Three studies reported healthcare providers' attitudes towards aspirin for cancer prevention (Smith et al., 2017; Chen et al., 2017; Das et al., 2008) (Table 3). All studies were of medium MMAT quality (3/5). Samples consisted of gastroenterologists (Chen et al., 2017; Das et al., 2008), genetics professionals (Chen et al., 2017), colorectal surgeons (Chen et al., 2017) and general practitioners (Smith et al., 2017). Two studies reported data on healthcare providers' attitudes towards the use of aspirin for patients at higher risk of cancer (Lynch syndrome, Barrett's oesophagus) (Chen et al., 2017; Das et al., 2008). In both studies, a high proportion of healthcare provider respondents $(72.0-76.0 \%)$ perceived aspirin to be a suitable cancer prevention option (Chen et al., 2017; Das et al., 2008).

A UK survey of general practitioners found willingness to prescribe aspirin was higher at lower doses, with $91.3 \%$ willing at $100 \mathrm{mg}, 81.8 \%$ willing at $300 \mathrm{mg}$, and $62.3 \%$ willing at $600 \mathrm{mg}$ (Smith et al., 2017). General practitioners were significantly more willing to prescribe aspirin at $600 \mathrm{mg}$ if they had $>10$ years' professional experience, were aged $\geq 50$, had greater awareness of the preventive effects of aspirin, and if they had seen a Lynch syndrome patient in clinic (range, odds ratio: 1.44 to 1.58) (Smith et al., 2017). There was evidence to suggest profession may influence willingness, with general practitioners who had a special interest in family history significantly less willing to prescribe aspirin (odds ratio: 0.41) (Smith et al., 2017). An Australian survey also found that a higher proportion of gastroenterologists (41/49, 83.7\%) and genetic professionals $(49 / 59,83.1 \%)$ perceived aspirin to be effective for cancer prevention, than colorectal surgeons (47/73, 64.4\%) (Chen et al., 2017). Across all three studies, we did not observe a pattern between year of study and healthcare providers' attitudes towards aspirin for preventive therapy (Smith et al., 2017; Chen et al., 2017; Das et al., 2008).

\subsection{Study quality}

We assessed methodological quality using the MMAT (Table 4). Twenty-five studies were quantitative RCTs (Logan et al., 2008; Rexrode et al., 2000; Hull et al., 2018; Jankowski et al., 2018; Cook et al., 2013; Duggan et al., 2014; Lipton et al., 1982; Roop et al., 2013; Roy et al., 2017; Sample et al., 2002a; Sandler et al., 2003; Liesenfeld et al., 2016; Benamouzig et al., 2001; Benamouzig et al., 2003; Benamouzig et al., 2012; Ishikawa et al., 2013; Baron et al., 2003; Burn et al., 2008; Burn et al., 2013; Falk et al., 2012; Pommergaard et al., 2016; Sample et al., 2002b; Garland et al., 2019; Joharatnam-Hogan et al., 2019; Sinicrope et al., 2019), 8 were quantitative descriptive studies (Yachimski et al., 2015; Jensen et al., 2016; Hur et al., 2009; Hur et al., 2008; Nguyen et al., 2019; Smith et al., 2017; Chen et al., 2017; Das et al., 2008), and five were quantitative non-randomised studies (Barnes et al., 1999; Burney et al., 1996; Frommel et al., 1997; Krishnan et al., 2001; Ruffin et al., 1997). No qualitative studies were identified. Of the RCTs, one study (4\%) scored 4/4 for quality (Hull et al., 2018), 36\% (9/25) scored 3/4 (Logan et al., 2008; Cook et al., 2013; Roy et al., 2017; Sandler et al., 2003; Ishikawa et al., 2013; Baron et al., 2003; Burn et al., 2013; Pommergaard et al., 2016; Joharatnam-Hogan et al., 2019), and 24\%
Table 4

Mixed Methods Appraisal Tool assessment for the 38 included studies.

\begin{tabular}{|c|c|c|c|c|c|c|c|}
\hline & \multirow[b]{2}{*}{$n$} & \multicolumn{2}{|l|}{ Yes } & \multicolumn{2}{|c|}{ No } & \multicolumn{2}{|c|}{$\begin{array}{l}\text { Cannot } \\
\text { tell }\end{array}$} \\
\hline & & $n$ & $\%$ & $n$ & $\%$ & $n$ & $\%$ \\
\hline $\begin{array}{l}\text { 2. Quantitative randomised } \\
\text { controlled trials }\end{array}$ & 25 & & & & & & \\
\hline $\begin{array}{l}\text { 2.1. Is randomization appropriately } \\
\text { performed? }\end{array}$ & & 6 & 24 & 0 & 0 & 19 & 76 \\
\hline $\begin{array}{l}\text { 2.2. Are the groups comparable at } \\
\text { baseline? }\end{array}$ & & 20 & 80 & 2 & 8 & 3 & 12 \\
\hline 2.3. Are there complete outcome data? & & 19 & 76 & 4 & 16 & 2 & 8 \\
\hline $\begin{array}{l}\text { 2.4. Are outcome assessors blinded to } \\
\text { the intervention provided? }\end{array}$ & & 10 & 40 & 4 & 16 & 11 & 44 \\
\hline $\begin{array}{l}\text { 3. Quantitative non-randomised } \\
\text { studies }\end{array}$ & 5 & & & & & & \\
\hline $\begin{array}{l}\text { 3.1. Are the participants' } \\
\text { representative of the target } \\
\text { population? }\end{array}$ & & 4 & 80 & 1 & 20 & 0 & 0 \\
\hline $\begin{array}{l}\text { 3.2. Are measurements appropriate } \\
\text { regarding both the outcome and } \\
\text { intervention (or exposure)? }\end{array}$ & & 5 & 100 & 0 & 0 & 0 & 0 \\
\hline 3.3. Are there complete outcome data? & & 4 & 80 & 1 & 20 & 0 & 0 \\
\hline $\begin{array}{l}\text { 3.4. Are the confounders accounted for } \\
\text { in the design and analysis? }\end{array}$ & & 0 & 0 & 1 & 20 & 4 & 80 \\
\hline $\begin{array}{l}\text { 3.5 During the study period, is the } \\
\text { intervention administered (or } \\
\text { exposure occurred) as intended? }\end{array}$ & & 3 & 60 & 1 & 20 & 1 & 20 \\
\hline 4. Quantitative descriptive studies & 8 & & & & & & \\
\hline $\begin{array}{l}\text { 4.1. Is the sampling strategy relevant to } \\
\text { address the research question? }\end{array}$ & & 4 & 50 & 3 & 38 & 1 & 13 \\
\hline $\begin{array}{l}\text { 4.2. Is the sample representative of the } \\
\text { target population? }\end{array}$ & & 1 & 13 & 5 & 63 & 2 & 25 \\
\hline $\begin{array}{l}\text { 4.3. Are the measurements } \\
\text { appropriate? }\end{array}$ & & 5 & 63 & 2 & 25 & 1 & 13 \\
\hline 4.4. Is the risk of nonresponse bias low? & & 4 & 50 & 1 & 13 & 3 & 38 \\
\hline $\begin{array}{l}\text { 4.5. Is the statistical analysis } \\
\text { appropriate to answer the research } \\
\text { question? }\end{array}$ & & 7 & 88 & 0 & 0 & 1 & 13 \\
\hline
\end{tabular}

(6/25) of studies met one criterion (Rexrode et al., 2000; Lipton et al., 1982; Sample et al., 2002a; Benamouzig et al., 2001; Benamouzig et al., 2003; Sample et al., 2002b). Of the quantitative non-randomised studies, two studies (40\%) scored 4/5 on the MMAT (Barnes et al., 1999; Krishnan et al., 2001), two studies (40\%) scored 3/5 (Frommel et al., 1997; Ruffin et al., 1997), and one study (20\%) scored 2/5 (Burney et al., 1996). Of the quantitative descriptive studies, 38\% (3/8) scored 2/5 on the MMAT (Yachimski et al., 2015; Hur et al., 2009; Hur et al., 2008), and 63\% (5/8) scored 3/5 (Jensen et al., 2016; Nguyen et al., 2019; Smith et al., 2017; Chen et al., 2017; Das et al., 2008).

\section{Discussion}

In this systematic review investigating attitudes and behaviour towards aspirin for preventive therapy, we found moderate to high levels of uptake to an aspirin clinical trial among people who were eligible to participate. A large proportion of participants in trials reported high levels of adherence on a day-to-day basis. At short-term follow up, most people were still taking aspirin for cancer prevention. However, there was mixed evidence observed for long-term persistence with aspirin. Given that aspirin is recommended to be taken regularly for several years for a cancer preventive benefit (Bibbins-Domingo, 2016; National Institute for Health and Clinical Excellence (NICE), 2020), persistence among users of aspirin should be investigated further.

In contrast to the more extensive behavioural research conducted in breast cancer preventive therapy (Hackett et al., 2018; Smith et al., 2016; Bober et al., 2004; Holmberg et al., 2017; Thorneloe et al., 2019; Rondanina et al., 2008), minimal research has examined the factors associated with use of aspirin for cancer prevention. In our review, we only identified four studies reporting any factors associated with 
adherence, and none with uptake. Additionally, no qualitative studies were identified. Several studies investigated willingness or intention to use aspirin, which was found to be moderately high among members of the public and those at higher cancer risk. The demographic, clinical and psychological factors associated with willingness and intentions were also investigated, but evidence was either limited or conflicting.

While observational studies were eligible, we only identified trials reporting uptake and adherence data, which presents generalisability issues. Trial participants may be more motivated to use aspirin than those in routine care, and frequent follow-ups may have increased adherence rates. Previous research has also observed that people at lower socioeconomic status (Gross et al., 2005) and those from an ethnic minority group (Du et al., 2006) are less likely to participate in cancer trials. Furthermore, the decision to participate in a trial would not have been just a consideration of aspirin, but also other agents being simultaneously investigated. The four trials reporting uptake data were also evaluating esomeprazole, vitamin $\mathrm{E}$, folate, and eicosapentaenoic acid alongside aspirin. Members of the public may be less familiar with these agents, which may have negatively affected their decision to participate in the trial.

In our review, we identified studies conducted across multiple decades (1982 to 2019). However, official guidance recommending the use of aspirin for colorectal cancer prevention has only recently been introduced (2016 onwards) (Bibbins-Domingo, 2016; National Institute for Health and Clinical Excellence (NICE), 2020; Cancer Council Australia, 2019). While we did not find an increase over time in trial uptake and adherence, future trials may observe higher rates of uptake and adherence as official guidance becomes more widely known among the public and healthcare providers. Furthermore, in the future we may observe an increasing trend in positive attitudes towards aspirin for preventive therapy.

Despite searching for studies using aspirin for secondary cancer prevention, most articles investigated aspirin for primary prevention. Our review findings should be applied with caution to a secondary prevention context. Patients who have previously had cancer may have different motivations for taking aspirin than those offered aspirin for primary prevention. Healthcare providers may also have less positive views towards aspirin for secondary cancer prevention, as a lower number of secondary prevention trials have been conducted compared with primary prevention (Langley et al., 2011). However, there is a large ongoing trial in the adjuvant setting (Add-Aspirin trial), which will provide further evidence on the effects of regular aspirin use in patients with non-metastatic breast, colorectal, gastro-oesophageal, and prostate cancer (Coyle et al., 2016).

Relevant studies have been published following our search cut-off date that contribute further to our knowledge in this topic area. Similar to our review findings, the ASPIRED trial, investigating aspirin for colorectal cancer prevention, found that most participants reported high levels of day-to-day adherence to aspirin at dose of $81 \mathrm{mg}(79 \%$ reported 95-100\% adherence) and $325 \mathrm{mg}$ (91\% reported $95-100 \%$ adherence) (Drew et al., 2020). Furthermore, a recent qualitative study was published exploring healthcare professionals' views on the Australian guidance recommending aspirin for colorectal cancer prevention for the public (Milton et al., 2021).

\subsection{Directions for future research}

Overall, we found that the likelihood that eligible users of aspirin would participate in a trial that requires randomization to aspirin for cancer prevention was between 40.9 and $77.7 \%$. Researchers developing a trial in this area should take these findings into consideration when planning and designing their study. While clinical guidelines in the US, Australia and the UK recommend aspirin for colorectal cancer prevention (Bibbins-Domingo, 2016; National Institute for Health and Clinical Excellence (NICE), 2020; Cancer Council Australia, 2019), it is currently unknown if people initiate and adhere to aspirin in routine care. To date, only studies reporting data on intentions and willingness to use aspirin have been published. As intentions do not always translate into behaviour (Sheeran and Webb, 2016), further research should investigate how people form a decision to initiate and adhere to aspirin for preventive therapy, and the support they may need.

Despite searching for studies investigating aspirin for any cancer prevention, the vast majority of identified studies focused on gastrointestinal cancer risk reduction. As the evidence base is stronger for gastrointestinal cancer prevention, we may expect lower rates of uptake, adherence and acceptability for other cancers (e.g. breast, lung, prostate). Research should investigate further rates of uptake and adherence of, and attitudes towards, aspirin for the prevention of nongastrointestinal cancers.

Previous research has found higher uptake of breast cancer preventive therapy among women with fewer concerns about its side-effects (Thorneloe et al., 2019; Rondanina et al., 2008). While there are several reported side-effects to using aspirin (Lanas and Scheiman, 2007; Cuzick et al., 2015), it is currently unknown the relationship between participants' side-effects, perceived or experienced, in relation to aspirin and their rates of uptake and adherence. We recommend that future research should investigate the relationship between these factors further.

The recent Australian qualitative study reported that healthcare providers viewed primary care physicians as having the most important role in the implementation of guidance recommending aspirin for cancer prevention (Milton et al., 2021). We recommend that future research aiming to examine decision-making in the context of aspirin for cancer prevention should focus on the primary care setting. In our review, we found moderately high levels of willingness among general practitioners to prescribe aspirin to patients with Lynch syndrome. Factors that may be influencing willingness include the aspirin dose, professional background, and awareness of the cancer preventive benefits of aspirin.

The review had limitations. Due to time and resource constraints, the literature was limited to English language articles, and second reviewers only duplicated screening, data extraction, and quality assessment for a proportion of articles (20-45\%). Our review excluded studies that did not use or prescribe aspirin for the primary purpose of cancer prevention, such as the ASPREE trial which had fatal and non-fatal cancer as a secondary endpoint (McNeil et al., 2018). However, in clinical practice consideration to use aspirin is likely to factor in both its use as a form of cancer preventive therapy and other outcomes, such as cardiovascular disease prevention. Uptake rates to a clinical trial were also strongly affected by the approach used to calculate uptake. For example, as reported in supplementary Table 1, when we calculated rates of uptake to a trial with the denominator all people who were approached about the trial, including those who were ineligible to participate, uptake rates were much lower. More standardised and transparent reporting of uptake data is warranted to compare across cohorts.

\section{Conclusions}

Overall, we found that most people who were eligible and offered participation in an aspirin trial accepted. The majority of participants also reported a good level of adherence on a day-to-day basis. We found high levels of short-term aspirin persistence, but evidence was mixed for long-term persistence. No studies examined uptake and adherence in routine care, and minimal research investigated the factors associated with using aspirin. Overall, we found that there is substantial scope for research into the barriers and facilitators to implementing aspirin for preventive therapy into clinical care.

\section{Collaborators}

This work is on behalf of the Aspirin for Cancer Prevention Group (AsCaP): Senior Executive Board Prof. J Burn, Prof. A.T Chan, Prof. J Cuzick, Dr. B Nedjai, Prof. Ruth Langley. 


\section{Funding}

This work was supported by Cancer Research UK (AsCaP) [grant number C569/A24991]. KEL is supported by an Economic and Social Research Council studentship [grant number ES/P000745/1]. This report is independent research supported by the National Institute for Health Research NIHR Advanced Fellowship, Dr. Samuel Smith [grant number NIHR300588]. SGS also acknowledges funding support from a Yorkshire Cancer Research University Academic Fellowship. The funders had no role in study design, data collection and analysis, decision to publish, or preparation of the manuscript.

\section{Disclosure}

RJT has received honorarium from Novartis. All remaining authors declare no conflicts of interest.

\section{Declaration of Competing Interest}

The authors declare that they have no known competing financial interests or personal relationships that could have appeared to influence the work reported in this paper.

\section{Appendix A. Supplementary data}

Supplementary data to this article can be found online at https://doi. org/10.1016/j.ypmed.2021.106872.

\section{References}

Barnes, C.J., Hamby-Mason, R.L., Hardman, W.E., et al., 1999. Effect of aspirin on prostaglandin $\mathrm{e} 2$ formation and transforming growth factor alpha expression in human rectal mucosa from individuals with a history of adenomatous polyps of the colon. Cancer Epidemiol. Biomark. Prev. 8 (4 Pt 1), 311-315.

Baron, J.A., Cole, B.F., Sandler, R.S., et al., 2003. A randomized trial of aspirin to prevent colorectal adenomas. N. Engl. J. Med. 348 (10), 891-899.

Benamouzig, R., Yoon, H., Little, J., et al., 2001. Apacc, a french prospective study on aspirin efficacy in reducing colorectal adenoma recurrence: design and baseline findings. Eur. J. Cancer Prev. 10 (4), 327-335.

Benamouzig, R., Deyra, J., Martin, A., et al., 2003. Daily soluble aspirin and prevention of colorectal adenoma recurrence: one-year results of the apacc trial. Gastroenterology 125 (2), 328-336.

Benamouzig, R., Uzzan, B., Deyra, J., et al., 2012. Prevention by daily soluble aspirin of colorectal adenoma recurrence: 4-year results of the apacc randomised trial. Gut 61 (2), 255-261.

Bibbins-Domingo, K., 2016. Aspirin use for the primary prevention of cardiovascular disease and colorectal cancer: U.S. preventive services task force recommendation statement. Ann. Intern. Med. 164 (12), 836-845.

Bober, S.L., Hoke, L.A., Duda, R.B., et al., 2004. Decision-making about tamoxifen in women at high risk for breast cancer: clinical and psychological factors. J. Clin. Oncol. 22 (24), 4951-4957.

Bosetti, C., Santucci, C., Gallus, S., et al., 2020. Aspirin and the risk of colorectal and other digestive tract cancers: an updated meta-analysis through 2019. Ann. Oncol. 31 (5), 558-568.

Bray, F., Ferlay, J., Soerjomataram, I., et al., 2018. Global cancer statistics 2018: GLOBOCAN estimates of incidence and mortality worldwide for 36 cancers in 185 countries. CA Cancer J. Clin. 68 (6), 394-424.

Burn, J., Bishop, D.T., Mecklin, J.P., et al., 2008. Effect of aspirin or resistant starch on colorectal neoplasia in the lynch syndrome. N. Engl. J. Med. 359 (24), 2567-2578.

Burn, J., Mathers, J.C., Bishop, D.T., 2013. Chemoprevention in lynch syndrome. Familial Cancer 12 (4), 707-718.

Burney, K.D., Krishnan, K., Ruffin, M.T., et al., 1996. Adherence to single daily dose of aspirin in a chemoprevention trial - an evaluation of self-report and microelectronic monitoring. Arch. Fam. Med. 5 (5), 297-300.

Cancer Council Australia, 2019. Chemopreventive Candidate Agents. Issued: January. Available from: https://wiki.cancer.org.au/australia/Clinical_question:Aspirin_for _prevention_of_colorectal_cancer. (Accessed March 2021).

Chen, Y., Peate, M., Kaur, R., et al., 2017. Exploring clinicians' attitudes about using aspirin for risk reduction in people with lynch syndrome with no personal diagnosis of colorectal cancer. Familial Cancer 16 (1), 99-109.

Cook, N.R., Lee, I.M., Zhang, S.M., et al., 2013. Alternate-day, low-dose aspirin and cancer risk: long-term observational follow-up of a randomized trial. Ann. Intern. Med. 159 (2), 77-85.

Coyle, C., Cafferty, F.H., Rowley, S., et al., 2016. ADD-ASPIRIN: a phase III, double-blind, placebo controlled, randomised trial assessing the effects of aspirin on disease recurrence and survival after primary therapy in common non-metastatic solid tumours. Contemp. Clin. Trials 51, 56-64.
Cuzick, J., Thorat, M.A., Bosetti, C., et al., 2015. Estimates of benefits and harms of prophylactic use of aspirin in the general population. Ann. Oncol. 26 (1), 47-57.

Das, D., Ishaq, S., Harrison, R., et al., 2008. Management of barrett's esophagus in the UK: Overtreated and underbiopsied but improved by the introduction of a national randomized trial. Am. J. Gastroenterol. 103 (5), 1079-1089.

Drew, D.A., Schuck, M.M., Magicheva-Gupta, M.V., et al., 2020. Effect of low-dose and standard-dose aspirin on PGE2 biosynthesis among individuals with colorectal adenomas: a randomized clinical trial. Cancer Prev. Res. 13 (10), 877-888.

Du, W., Gadgeel, S.M., Simon, M.S., 2006. Predictors of enrollment in lung cancer clinical trials. Cancer 106 (2), 420-425.

Duggan, C., Wang, C.Y., Xiao, L., et al., 2014. Aspirin and serum estrogens in postmenopausal women: a randomized controlled clinical trial. Cancer Prev. Res. 7 (9), 906-912.

Falk, G.W., Buttar, N.S., Foster, N.R., et al., 2012. A combination of esomeprazole and aspirin reduces tissue concentrations of prostaglandin e-2 in patients with barrett's esophagus. Gastroenterology 143 (4), 917-926.

Frommel, T.O., Dyavanapalli, M., Oldham, T., et al., 1997. Effect of aspirin on prostaglandin e2 and leukotriene b4 production in human colonic mucosa from cancer patients. Clin. Cancer Res. 3 (2), 209-213.

Furlan, A.D., Pennick, V., Bombardier, C., et al., 2009. 2009 updated method guidelines for systematic reviews in the Cochrane Back Review Group. Spine 34 (18), 1929-1941.

Garland, L.L., Guillen-Rodriguez, J., Hsu, C.H., et al., 2019. Effect of intermittent versus continuous low-dose aspirin on nasal epithelium gene expression in current smokers: a randomized, double-blinded trial. Cancer Prev. Res. 12 (11), 809-820.

Gross, C.P., Filardo, G., Mayne, S.T., et al., 2005. The impact of socioeconomic status and race on trial participation for older women with breast cancer. Cancer 103 (3), 483-491.

Hackett, J., Thorneloe, R., Side, L., et al., 2018. Uptake of breast cancer preventive therapy in the UK: results from a multicentre prospective survey and qualitative interviews. Breast Cancer Res. Treat. 170 (3), 633-640.

Holmberg, C., Bandos, H., Fagerlin, A., et al., 2017. NRG oncology/national surgical adjuvant breast and bowel project decision-making project-1 results: decision making in breast cancer risk reduction. Cancer Prev. Res. 10 (11), 625-634.

Hull, M.A., Sprange, K., Hepburn, T., et al., 2018. Eicosapentaenoic acid and aspirin, alone and in combination, for the prevention of colorectal adenomas (seAFOod Polyp Prevention trial): a multicentre, randomised, double-blind, placebocontrolled, 2 x 2 factorial trial. Lancet 392 (10164), 2583-2594.

Hur, C., Broughton, D.E., Ozanne, E., et al., 2008. Patient preferences for the chemoprevention of esophageal adenocarcinoma in barrett's esophagus. Am. J. Gastroenterol. 103 (10), 2432-2442.

Hur, C., Broughton, D.E., Kong, C.Y., et al., 2009. Patient preferences for the chemoprevention of colorectal cancer. Dig. Dis. Sci. 54 (10), 2207-2214.

Hurwitz, L.M., Michels, K.A., Cook, M.B., et al., 2021. Associations between daily aspirin use and cancer risk across strata of major cancer risk factors in two large U.S. cohorts. Cancer Causes Control 32 (1), 57-65.

Ishikawa, H., Wakabayashi, K., Suzuki, S., et al., 2013. Preventive effects of low-dose aspirin on colorectal adenoma growth in patients with familial adenomatous polyposis: double-blind, randomized clinical trial. Cancer Med. 2 (1), 50-56.

Jankowski, J.A.Z., de Caestecker, J., Love, S.B., et al., 2018. Esomeprazole and aspirin in Barrett's oesophagus (AspECT): a randomised factorial trial. Lancet 392 (10145), 400-408.

Jensen, J.D., Holton, A.E., Krakow, M., et al., 2016. Colorectal cancer prevention and intentions to use low-dose aspirin: a survey of 1000 u.S. Adults aged 40-65. Cancer Epidemiol. 41, 99-105.

Jiang, Y., Su, Z., Li, C., et al., 2021. Association between the use of aspirin and risk of lung cancer: results from pooled cohorts and Mendelian randomization analyses. J. Cancer Res. Clin. Oncol. 147 (1), 139-151.

Joharatnam-Hogan, N., Cafferty, F., Hubner, R., et al., 2019. Aspirin as an adjuvant treatment for cancer: feasibility results from the add-aspirin randomised trial. Lancet Gastroenterol. Hepatol. 4 (11), 854-862.

Krishnan, K., Ruffin, M.T., Normolle, D., et al., 2001. Colonic mucosal prostaglandin e2 and cyclooxygenase expression before and after low aspirin doses in subjects at high risk or at normal risk for colorectal cancer. Cancer Epidemiol. Biomark. Prev. 10 (5), 447-453.

Lanas, A., Scheiman, J., 2007. Low-dose aspirin and upper gastrointestinal damage: epidemiology, prevention and treatment. Curr. Med. Res. Opin. 23 (1), 163-173.

Langley, R.E., Burdett, S., Tierney, J.F., et al., 2011. Aspirin and cancer: has aspirin been overlooked as an adjuvant therapy? Br. J. Cancer 105 (8), 1107-1113.

Liesenfeld, D.B., Botma, A., Habermann, N., et al., 2016. Aspirin reduces plasma concentrations of the oncometabolite 2-hydroxyglutarate: results of a randomized, double-blind, crossover trial. Cancer Epidemiol. Biomark. Prev. 25 (1), 180-187.

Lipton, A., Scialla, S., Harvey, H., et al., 1982. Adjuvant antiplatelet therapy with aspirin in colo-rectal cancer. J. Med. 13 (5-6), 419-429.

Logan, R.F., Grainge, M.J., Shepherd, V.C., et al., 2008. Aspirin and folic acid for the prevention of recurrent colorectal adenomas. Gastroenterology 134 (1), 29-38.

McNeil, J.J., Nelson, M.R., Woods, R.L., et al., 2018. Effect of aspirin on all-cause mortality in the healthy elderly. N. Engl. J. Med. 379 (16), 1519-1528.

Milton, S., McIntosh, J., Yogaparan, T., et al., 2021. Clinicians' opinions on recommending aspirin to prevent colorectal cancer to Australians aged 50-70 years: a qualitative study. BMJ Open 11 (2), e042261.

Moher, D., Liberati, A., Tetzlaff, J., et al., 2009. Preferred reporting items for systematic reviews and meta-analyses: the PRISMA statement. Ann. Intern. Med. 151 (4), 264-269. 
Naghavi, M., Abajobir, A.A., Abbafati, C., et al., 2017. Global, regional, and national agesex specific mortality for 264 causes of death, 1980-2016: a systematic analysis for the Global Burden of Disease Study 2016. Lancet 390 (10100), 1151-1210.

National Institute for Health and Clinical Excellence (NICE), 2020. Colorectal Cancer [NG151]. Issued: January. Available from: https://www.nice.org.uk/guida nce/NG151. (Accessed December 2020).

Nguyen, P., McIntosh, J., Bickerstaffe, A., et al., 2019. Benefits and harms of aspirin to reduce colorectal cancer risk: a cross-sectional study of methods to communicate risk in primary care. Br. J. Gen. Pract. 69 (689), e843-e849.

Pace, R., Pluye, P., Bartlett, G., et al., 2012. Testing the reliability and efficiency of the pilot Mixed Methods Appraisal Tool (MMAT) for systematic mixed studies review. Int. J. Nurs. Stud. 49 (1), 47-53.

Petticrew, M., Roberts, H., 2006. Systematic Reviews in the Social Sciences: A Practical Guide. Blackwell, Oxford.

Pluye, P., Hong, Q.N., 2014. Combining the power of stories and the power of numbers: mixed methods research and mixed studies reviews. Annu. Rev. Public Health 35, $29-45$.

Pommergaard, H.C., Burcharth, J., Rosenberg, J., et al., 2016. Aspirin, calcitriol, and calcium do not prevent adenoma recurrence in a randomized controlled trial. Gastroenterology 150 (1), 114-122.

Rexrode, K.M., Lee, I.M., Cook, N.R., et al., 2000. Baseline characteristics of participants in the women's health study. J. Womens Health Gend. Based Med. 9 (1), 19-27.

Rondanina, G., Puntoni, M., Severi, G., et al., 2008. Psychological and clinical factors implicated in decision making about a trial of low-dose tamoxifen in hormone replacement therapy users. J. Clin. Oncol. 26 (9), 1537-1543.

Roop, R.P., Naughton, M.J., Van Poznak, C., et al., 2013. A randomized phase II trial investigating the effect of platelet function inhibition on circulating tumor cells in patients with metastatic breast cancer. Clin. Breast Cancer 13 (6), 409-415.

Rothwell, P.M., Wilson, M., Elwin, C.E., et al., 2010. Long-term effect of aspirin on colorectal cancer incidence and mortality: 20-year follow-up of five randomised trials. Lancet 376 (9754), 1741-1750.

Rothwell, P.M., Fowkes, F.G.R., Belch, J.F., et al., 2011. Effect of daily aspirin on long term risk of death due to cancer: analysis of individual patient data from randomised trials. Lancet 377 (9759), 31-41.

Roy, H.K., Turzhitsky, V., Wali, R., et al., 2017. Spectral biomarkers for chemoprevention of colonic neoplasia: a placebo-controlled double-blinded trial with aspirin. Gut 66 (2), 285-292.

Ruffin, M.T., Krishnan, K., Rock, C.L., et al., 1997. Suppression of human colorectal mucosal prostaglandins: determining the lowest effective aspirin dose. J. Natl. Cancer Inst. 89 (15), 1152-1160.
Ryan, R., Cochrane Consumers and Communication Review Group, 2016. Heterogeneity and Subgroup Analyses in Cochrane Consumers and Communication Group Reviews: Planning the Analysis at Protocol Stage. Issued: December. Available from: http://cccrg.cochrane.org. (Accessed August 2021).

Sample, D., Wargovich, M., Fischer, S.M., et al., 2002a. A dose-finding study of aspirin for chemoprevention utilizing rectal mucosal prostaglandin e-2 levels as a biomarker. Cancer Epidemiol. Biomark. Prev. 11 (3), 275-279.

Sample, D.A., Sinicrope, P.S., Wargovich, M.J., et al., 2002b. Post-study aspirin intake and factors motivating participation in a colorectal cancer chemoprevention trial. Cancer Epidemiol. Biomark. Prev. 11 (3), 281-285.

Sandler, R.S., Halabi, S., Baron, J.A., et al., 2003. A randomized trial of aspirin to prevent colorectal adenomas in patients with previous colorectal cancer. N. Engl. J. Med. 348 (10), 883-890.

Shea, B.J., Reeves, B.C., Wells, G., et al., 2017. AMSTAR 2: a critical appraisal tool for systematic reviews that include randomised or non-randomised studies of healthcare interventions, or both. BMJ 358, j4008.

Sheeran, P., Webb, T.L., 2016. The intention-behavior gap. Soc. Personal. Psychol. Compass 10 (9), 503-518.

Sinicrope, F.A., Velamala, P.R., Song, L., et al., 2019. Efficacy of difluoromethylornithine and aspirin for treatment of adenomas and aberrant crypt foci in patients with prior advanced colorectal neoplasms. Cancer Prev. Res. 12 (11), 821-830.

Smith, S.G., Sestak, I., Forster, A., et al., 2016. Factors affecting uptake and adherence to breast cancer chemoprevention: a systematic review and meta-analysis. Ann. Oncol. 27 (4), 575-590.

Smith, S.G., Foy, R., McGowan, J., et al., 2017. General practitioner attitudes towards prescribing aspirin to carriers of lynch syndrome: findings from a national survey. Familial Cancer 16 (4), 509-516.

Steward, W.P., Brown, K., 2013. Cancer chemoprevention: a rapidly evolving field. Br. J. Cancer 109 (1), 1-7.

Thorneloe, R.J., Horne, R., Side, L., et al., 2019. Beliefs about medication and uptake of preventive therapy in women at increased risk of breast cancer: results from a multicenter prospective study. Clin. Breast Cancer 19 (1), e116-e126.

Vrijens, B., De Geest, S., Hughes, D.A., et al., 2012. A new taxonomy for describing and defining adherence to medications. Br. J. Clin. Pharmacol. 73 (5), 691-705.

Yachimski, P., Wani, S., Givens, T., et al., 2015. Preference of endoscopic ablation over medical prevention of esophageal adenocarcinoma by patients with barrett's esophagus. Clin. Gastroenterol. Hepatol. 13 (1), 84-90. 